九州大学学術情報リポジトリ

Kyushu University Institutional Repository

\title{
Seasonal Changes in the Concentrations of Terpenic Compounds Contained in the Leaves of Coniferous Trees
}

SHIN, Changseob

Department of Forest Science, Chungbuk National University

LEE, Jeongwon

National Center for Forest Therapy

YEON, Poungsik

National Center for Forest Therapy

CHA, Joo Young

Field Science Center for Northern Biosphere, Hokkaido University

他

https://doi.org/10.5109/2231629

出版情報：九州大学大学院農学研究院紀要. 64 (1)，pp.15-26，2019-02-28. Faculty of Agriculture， Kyushu University

バージョン:

権利関係： 


\title{
Seasonal Changes in the Concentrations of Terpenic Compounds Contained in the Leaves of Coniferous Trees
}

\author{
Changseob SHIN ${ }^{1}$, Jeongwon LEE $^{2}$, Poungsik YEON ${ }^{2 *}$ \\ Joo Young $\mathrm{CHA}^{3}$ and Shoji OHGA \\ Laboratory of Forest Recources Management, Division of Forest Environmental Sciences, \\ Department of Ago-environmental Sciences, Faculty of Agriculture, \\ Kyushu University, Fukuoka, 811-2415, Japan \\ (Received October 18, 2018 and accepted November 12, 2018)
}

\begin{abstract}
This research tries to compare terpene compounds of five species of coniferous trees to find out which tree has relatively high-concentrated terpene compounds, and to check their concentration by season to find out which season the concentration is the highest. In addition, it tries to find out whether terpene compounds in the leaves are actually volatilized into the atmosphere. The results of the research show that there were no relatively high-concentrated terpene compounds in the leaves of Pinus densiflora and Pinus koraiensis, compared to the others. However, when the concentration of terpene compounds, which are volatilized in an airtight container, was checked, relatively high-concentrated terpene compounds were volatilized: 8 kinds of terpene compounds in the leaves of Pinus densiflora; 16 in Pinus koraiensis; 10 in Abies holophylla; 1 in Chamaecyparis obtusa; 2 in Chamaecyparis pisifera. And the concentration of terpene compounds by season was checked, the concentration of 21 terpene compounds was high in spring, excluding those of Abies holophylla, which showed high concentration in summer. The concentration of the terpene compounds by substance was generally high in spring and summer. Therefore, it is more effective to extract essential oil from coniferous trees in spring and summer.
\end{abstract}

Key words: Terpene compound, Essential oil, Softwood, Season

\section{INTRODUCTION}

As South Korea has become economically affluent and the people's average life expectancy has been extended, the people's interest in healthy lifestyles has become very high. In addition, the people's interest in comfortable environments and forests that can support a healthy lifestyle also increased and populations that go to mountains have been rapidly increasing. One important reason for going to forests as such is that the people believe that the volatile organic compounds (phytoncide) that volatilize from plants will enhance immune functions and health functions. Many studies on phytoncide have already been conducted.

The phytoncides that volatilize from Pinus densiflora, Pinus koraiensis, Chamaecyparis obtusa, and Chamaecyparis pisifera act on the central nervous system and thus are effective for stress relief ( $\mathrm{Na}$ et al., 1998, 1999). In particular, the essential oil of Chamaecyparis obtusa leaves has been reported as having antibacterial effects (Lee et al., 2001), antifungal effects (Lee et al., 2001; Park et al., 2005; Gwak et al., 2006), antimicrobial effects (Kang et al., 2007), effects to reduce the concentration of immunoglobulin-E that causes atopy (Korea Forest Service 2014), and antioxi-

\footnotetext{
1 Department of Forest Science, Chungbuk National University, Cheongju, 28644, Korea

2 National Center for Forest Therapy, Yeongju, 36043, Republic of Korea

${ }^{3}$ Field Science Center for Northern Biosphere, Hokkaido University, Sapporo 060-0809, Japan

* Corresponding author (E-mail: imoscow@hanmail.net)
}

dative effects (Park et al., 2008). In addition, it has also been reported as having effects to deodorize formaldehyde, which can play the role of a sick house syndrometreating agent (Kim et al., 2009), hair growth promoting effects (Park et al., 2013), human stress index and fatigue index reducing effects (Shin et al., 2010), and human depression-relieving effects (Lee et al., 2011). As studies and mass communication promotions have been concentrated on Chamaecyparis obtuse as such, Chamaecyparis obtuse has been magnified as the most preferred afforestation tree species and a tendency to regard other tree species as relatively less valuable has been formed.

However, not only Chamaecyparis obtuse but also the essential oils of other coniferous trees, such as Pinus koraiensis, Pinus densiflora, Chamaecyparis obtusa, Abies koreana E.H. Wilson, Abies holophylla, and Picea jezoensis (S. et Z.) Carriere, were also found to be commonly containing $\alpha$-pinene, camphene, myrcene, limonene, and bornyl acetate (Moon and Yoo, 2005). As such, terpenic compounds contained in coniferous trees' essential oils were reported as having biological properties, such as anti-inflammatory properties, bactericidal properties, appetite improving properties, tonic properties, circulation promoting properties, deodorizing properties, phlegm discharging properties, pesticidal properties, and tranquilizing properties (Mishra and Dubey, 1994).

Therefore, the present study was intended to extract seasonally essential oils from the leaves of five coniferous tree species, including Chamaecyparis obtuse, to compare changes in terpenic compounds, and to identify the correlations between leaf terpenic compound con- 
tents and the quantities of terpenic compounds volatized to the air.

\section{MATERIALS AND METHODS}

\section{Determination of materials to be analyzed}

As the purpose of the present study was to determine the terpenic compound contents in Pinus densiflora, Pinus koraiensis, Abies holophylla, Chamaecyparis obtusa, and Chamaecyparis pisifera, 21 kinds of terpenic compounds known to be useful to humans were selected as study subjects based on Chamaecyparis obtuse-related studies (Lee, 1999; Park et al., 2008; Kim et al., 2009; Kim et al., 2011; Korea Forest Service, 2014) and other coniferous tree-related studies (Kubeczka and Schultze, 1987; Moon and Yoo, 2005; Bakkali et al., 2008; Oh et al., 2013; Kim, 2014). In addition, for quantitative analyses, calibration curves for three concentrations of standard materials within the quantitation range were prepared (Table 1).

\section{Essential oil extraction and analysis}

Testing Materials

The tested tree species in the present study were coniferous trees comprising Pinus densiflora, Pinus koraiensis, Abies holophylla, Chamaecyparis obtusa, and Chamaecyparis pisifera and leaves were collected from three trees per species. As seasonal samples, Chamaecyparis obtusa samples were collected in Jagye-ri, Yonghwa-myeon, and Chungcheongbuk-do and Pinus densiflora, Pinus koraiensis, Abies holophylla, and Chamaecyparis pisifera samples were collected in the academic forest of Chungbuk National University in Songgye-ri, Hansu-myeon, Jecheon-si, and Chungcheongbuk-do on May 21, 2014 (spring), July 30, 2014 (summer), October 22, 2014 (autumn), and January 29, 2015 (winter).

\section{Essential Oil Extraction and GC/MSD Analysis Using S.D.E Equipment}

After sampling, the samples were put into zipper bags $(30 \mathrm{~cm} \times 35 \mathrm{~cm}$, Clean Zipper Bag, Korea) and the zipper bags were sealed and put into ice boxes (ice box XX large number 3, Minseon General Trading Company, Korea) before being transported. Only green leaf parts of the sample were cut and ground with a mixer (HMF3250S, Hanil Mixer, Korea). Then, $100 \mathrm{~g}$ of the sample and 1,000 mL of distilled water were put into a 3,000-mL distillation flask and heated. Thereafter, $100 \mathrm{~mL}$ of diethyl ether (case no. 60-29-7, Merck, Germany) and $100 \mathrm{~mL}$ of pentane (case no. 1009-66-0, Junsei, Japan) were put into a 1,000-mL flask for collection to extract essential oil components for $5 \mathrm{~h}$ using simultaneous steam distillation equipment (S.D.E). Anhydrous sodium sul-

Table 1. The Calibration curve of standard materials

\begin{tabular}{|c|c|c|c|c|c|c|c|c|}
\hline \multirow{3}{*}{ Standards } & \multicolumn{6}{|c|}{ For essential oil } & \multirow{2}{*}{\multicolumn{2}{|c|}{$\begin{array}{c}\text { For sampling of terpene } \\
\text { emission }\end{array}$}} \\
\hline & \multicolumn{2}{|r|}{ May } & \multicolumn{2}{|r|}{ July } & \multicolumn{2}{|r|}{ October } & & \\
\hline & $\mathrm{R}^{2}$ & Standard curve & $\mathrm{R}^{2}$ & Standard curve & $\mathrm{R}^{2}$ & Standard curve & $\mathrm{R}^{2}$ & Standard curve \\
\hline isoprene & 0.9968 & $\mathrm{y}=59059 \mathrm{x}-32784$ & 0.9993 & $y=42462 x-65510$ & 0.9994 & $\mathrm{y}=67401 \mathrm{x}-18789$ & 0.9914 & $y=3782.6 x+77842$ \\
\hline bornyl acetate & 0.9996 & $y=112798 x+18528$ & 0.9984 & $y=128235 x-36249$ & 0.9987 & $y=186884 x-55125$ & 0.9983 & $y=8361.4 x-61394$ \\
\hline camphene & 1.0000 & $y=149417 x+2592.6$ & 0.999 & $y=134396 x-46494$ & 0.9999 & $\mathrm{y}=192209 \mathrm{x}-16469$ & 0.9984 & $y=10928 x-51315$ \\
\hline camphor & 0.9996 & $\mathrm{y}=129189 \mathrm{x}+10210$ & 0.9985 & $\mathrm{y}=145631 \mathrm{x}-46029$ & 0.9991 & $y=205249 x-43210$ & 0.9975 & $y=8788.9 x-64244$ \\
\hline carene & 0.9999 & $\mathrm{y}=147621 \mathrm{x}-2562.2$ & 0.9986 & $y=137418 x-56277$ & 0.9999 & $y=193566 x-25540$ & 0.9982 & $\mathrm{y}=13691 \mathrm{x}-78189$ \\
\hline eucalyptol & 0.9999 & $y=177460 x+4747$ & 0.9986 & $y=188003 x-65850$ & 0.9995 & $y=282325 x-39669$ & 0.9981 & $y=2746.3 x-10709$ \\
\hline limonene & 1.0000 & $\mathrm{y}=170970 \mathrm{x}-6133.8$ & 0.9985 & $y=162438 x-69222$ & 0.9998 & $y=227434 x-26540$ & 0.9976 & $y=14357 x-109440$ \\
\hline linalool & 0.9994 & $y=108335 x-25287$ & 0.9991 & $y=110433 x-25391$ & 0.9976 & $\mathrm{y}=160771 \mathrm{x}-75918$ & 0.9968 & $y=6228.5 x-69082$ \\
\hline myrcene & 0.9971 & $y=22916 x-13221$ & 0.9979 & $y=19904 x-10683$ & 0.9974 & $y=30074 x-15580$ & 0.9998 & $y=1930.6 x-4988.7$ \\
\hline p-cymene & 0.9999 & $\mathrm{y}=196934 \mathrm{x}+8310.2$ & 0.9986 & $y=200127 x-74063$ & 0.9999 & $y=283206 x-25644$ & 0.9983 & $y=31864 x-89386$ \\
\hline sabinene & 0.9999 & $y=149276 x-10282$ & 0.998 & $\mathrm{y}=137927 \mathrm{x}-67930$ & 0.9998 & $y=188280 x-33518$ & ND & \\
\hline terpineol & 1.0000 & $y=67024 x-2231.4$ & 0.9975 & $y=74167 x-22539$ & 0.9983 & $y=107343 x-33252$ & 0.9970 & $y=1718.9 x-16130$ \\
\hline terpinolene & 0.9998 & $\mathrm{y}=156450 \mathrm{x}-3130.2$ & 0.9981 & $y=159006 x-73916$ & 0.9995 & $y=223082 x-56497$ & 0.9980 & $\mathrm{y}=750.07 \mathrm{x}-4781.6$ \\
\hline$\alpha$-phellandrene & 0.9999 & $\mathrm{y}=171917 \mathrm{x}-3171.4$ & 0.9983 & $\mathrm{y}=160757 \mathrm{x}-75084$ & 0.9997 & $y=223433 x-43083$ & 0.9982 & $\mathrm{y}=20330 \mathrm{x}-108049$ \\
\hline$\alpha$-pinene & 1.0000 & $y=165112 x+4413$ & 0.999 & $y=144316 x-50260$ & 0.9997 & $y=202918 x-4430.8$ & 0.9986 & $\mathrm{y}=17440 \mathrm{x}-78742$ \\
\hline$\alpha$-terpinene & 0.9999 & $y=176196 x-5102$ & 0.9982 & $\mathrm{y}=170544 \mathrm{x}-77255$ & 0.9997 & $y=235954 x-30308$ & 0.9984 & $y=11553 x-59690$ \\
\hline$\beta$-pinene & 1.0000 & $\mathrm{y}=209379 \mathrm{x}+279.4$ & 0.9987 & $y=192011 x-74653$ & 0.9999 & $\mathrm{y}=270414 \mathrm{x}-25354$ & 0.9977 & $y=17589 x-127067$ \\
\hline$\gamma$-terpinene & 0.9999 & $y=170646 x-900.8$ & 0.9982 & $y=167945 x-75394$ & 0.9998 & $y=239569 x-41997$ & 0.9976 & $y=16144 x-135152$ \\
\hline cedrol & 0.9993 & $\mathrm{y}=113371 \mathrm{x}+13755$ & 0.9975 & $y=114465 x-45652$ & 1.0000 & $y=161346 x-208.6$ & 0.9967 & $\mathrm{y}=6227.7 \mathrm{x}-72966$ \\
\hline transcaryophyllene & 0.9992 & $y=114682 x+29267$ & 0.9983 & $y=131980 x-61809$ & 0.9975 & $y=191379 x-96382$ & 0.9983 & $y=4144 x-30037$ \\
\hline$\alpha$-humulene & 0.9995 & $\mathrm{y}=100661 \mathrm{x}-943.6$ & 0.9977 & $y=112863 x-56548$ & 0.9977 & $y=160076 x-80522$ & 0.9983 & $\mathrm{y}=11773 \mathrm{x}-83379$ \\
\hline
\end{tabular}


Table 2. GC/MSD conditions

\begin{tabular}{|c|c|c|c|c|c|}
\hline GC-MSD & \multicolumn{5}{|c|}{ GCMS-QP2010 Plus, Shimadzu, Japan } \\
\hline Carrier Gas & \multicolumn{5}{|c|}{ Helium (99.999) } \\
\hline Injector Temp. & \multicolumn{5}{|l|}{$220^{\circ} \mathrm{C}$} \\
\hline Interface Temp. & \multicolumn{5}{|l|}{$230^{\circ} \mathrm{C}$} \\
\hline Capillary & \multicolumn{5}{|c|}{ HP-INNOWAX } \\
\hline column & \multicolumn{5}{|c|}{$(30 \mathrm{~m} \times 0.25 \mathrm{mmI} . \mathrm{D} \times 0.25 \mu \mathrm{m}$, filmthickness $)$} \\
\hline \multirow[t]{2}{*}{ Column Flow } & \multicolumn{5}{|l|}{$0.9 \mathrm{~mL} / \mathrm{min}$} \\
\hline & Initial & Initial & Rate & Final & Final \\
\hline \multirow[t]{2}{*}{ Oven Program } & Temp. $\left({ }^{\circ} \mathrm{C}\right)$ & Time (min) & $\left({ }^{\circ} \mathrm{C} / \mathrm{min}.\right)$ & Temp. $\left({ }^{\circ} \mathrm{C}\right)$ & Time (min) \\
\hline & 35 & 6 & 5 & 240 & 2 \\
\hline Post run & \multicolumn{5}{|c|}{$250^{\circ} \mathrm{C}, 3 \mathrm{~min}$} \\
\hline Ion Source Temp. & \multicolumn{5}{|l|}{$250^{\circ} \mathrm{C}$} \\
\hline EI voltage & \multicolumn{5}{|l|}{$70 \mathrm{eV}$} \\
\hline Monitor Ion & \multicolumn{5}{|c|}{$\mathrm{m} / \mathrm{z}=35 \sim 400$} \\
\hline Solvent Delay & \multicolumn{5}{|l|}{$1 \mathrm{~min}$} \\
\hline Monitering mode & \multicolumn{5}{|l|}{ SCAN mode } \\
\hline
\end{tabular}

fate (case no. 7757-82-6, Junsei, Japan) was put into the extracted essential oil and the essential oil was kept in a $-2^{\circ} \mathrm{C}$ cold room for $24 \mathrm{~h}$ to agglutinate moisture. Thereafter, to remove the sodium sulfate that was holding in the moisture, the essential oil was pushed through a syringe (Kovax-syringe $50 \mathrm{~mL}$, Korea Vaccine Co., Ltd., Korea) installed with a filtration filter $(0.45 \mu \mathrm{m}$ syringe filter, Sartorius, Germany) to separate the dehydrated essential oil. The dehydrated essential oil was put into a $500-\mathrm{mL}$ round flask, the solvent in the essential oil was removed, the essential oil was being concentrated using a rotary decompression and compression system (CVE3100, Eyela, Japan), and the essential oil was moved to a 15-mL conical tube. To collect the essential oil sticking to the wall of the 500-mL round flask, $5 \mathrm{~mL}$ of diethyl ether (case no. 60-29-7, Merck, Germany) and $5 \mathrm{~mL}$ of pentane (case No. 1009-66-0, Junsei, Japan) were put into the flask. The wall was washed by micro-vibrating the flask using an ultrasonic washer (SD-D300H, Seongdong Ultrasonic Co., Korea), the collected essential oil was put into the abovementioned $15-\mathrm{mL}$ conical tube containing the essential oil, the essential oil was completely concentrated using a nitrogen evaporator (MG-2100, Eyela, Japan), and the weight of the concentrated essential oil was measured.

Thereafter, $100 \mu \mathrm{g}$ of the essential oil was taken and put into a $100-\mathrm{mL}$ volume flask and the flask was filled with dichloromethane (case no. 75-09-2, Sigma-Aldrich, USA) up to the scale mark to dilute the essential oil. Then, a certain quantity of the diluted essential oil was taken and used as a sample for GC/MSD analysis. GC/ MSD (GCMS-QP2010 Plus, Shimadzu, Japan) analysis conditions were set as shown in (Table 2) to identify terpenic compounds.

\section{Collection and Analysis of Terpenic Compounds Volatilizing from Coniferous Tree Leaves in Airtight Containers}

To analyze the correlation between the terpenic compounds contained in the leaves and the terpenic compounds volatilizing through stomas, the sample was put into an airtight container and the air in the container was collected and analyzed. Out of the sample (leaves) collected on October 22, $5.0 \mathrm{~g}$ was put into a plastic airtight container (Cereal dispenser 3.9 L, Lock \& Lock, China) and the air in the airtight container was collected with a sampling pump (MP- $\sum 30 \mathrm{KN}$, Sibata, Japan) installed with a solid absorption tube (KT50601, Supelco, USA) filled with Tenax-TA. The sample that was put into the airtight container together with the sampling pump and was maintained in a $20^{\circ} \mathrm{C}$ constant temperature state and the sampling pump was set to collect $2 \mathrm{~L}$ of the air from $90 \mathrm{~min}$ after sealing at a flow rate of

Table 3. Thermal desorber conditions

\begin{tabular}{ll}
\hline Thermal Desorber & $\begin{array}{l}\text { TurboMatrix ATD 350, } \\
\text { Perkinelmer, USA }\end{array}$ \\
\hline Column Flow & $1 \mathrm{~mL} / \mathrm{min}$ \\
Desorb Flow & $50 \mathrm{~mL} / \mathrm{min}$ \\
Temp. & Max. $320^{\circ} \mathrm{C}$ \\
Valve Temp. & $250^{\circ} \mathrm{C}$ \\
Transferline Temp. & $250^{\circ} \mathrm{C}$ \\
Cold trap & Tenax $-\mathrm{TA}$ \\
Low Temp. & $-30^{\circ} \mathrm{C}$ \\
High Temp. & $310^{\circ} \mathrm{C}$ \\
Rate & $40^{\circ} \mathrm{C} / \mathrm{sec}$ \\
Inlet Flow & off \\
Outlet Split ratio & $100: 1$
\end{tabular}


Table 4. GC/MSD conditions

\begin{tabular}{|c|c|c|c|c|c|}
\hline GC-MSD & \multicolumn{5}{|c|}{ GCMS-QP2010 Plus, Shimadzu, Japan } \\
\hline Carrier Gas & \multicolumn{5}{|c|}{ Helium (99.999) } \\
\hline Interface Temp. & \multicolumn{5}{|l|}{$230^{\circ} \mathrm{C}$} \\
\hline Capillary column & \multicolumn{5}{|c|}{ HP-INNOWAX $(30 \mathrm{~m} \times 0.25 \mathrm{mmI} . \mathrm{D} \times 0.25 \mu \mathrm{m}$, filmthickness $)$} \\
\hline Column Flow & \multicolumn{5}{|l|}{$1 \mathrm{~mL} / \mathrm{min}$} \\
\hline \multirow[t]{3}{*}{ Oven Program } & Initial & Initial & Rate & Final & Final \\
\hline & Temp. $\left({ }^{\circ} \mathrm{C}\right)$ & Time (min) & $\left({ }^{\circ} \mathrm{C} / \mathrm{min}.\right)$ & Temp. $\left({ }^{\circ} \mathrm{C}\right)$ & Time (min) \\
\hline & 35 & 6 & 5 & 240 & 2 \\
\hline Post run & \multicolumn{5}{|l|}{$250^{\circ} \mathrm{C}, 3 \mathrm{~min}$} \\
\hline Ion Source Temp. & \multicolumn{5}{|l|}{$250^{\circ} \mathrm{C}$} \\
\hline EI voltage & \multicolumn{5}{|l|}{$70 \mathrm{eV}$} \\
\hline Solvent Delay & \multicolumn{5}{|l|}{$1 \mathrm{~min}$} \\
\hline Monitering mode & \multicolumn{5}{|l|}{ SIM mode } \\
\hline
\end{tabular}

$100 \mathrm{~mL} / \mathrm{min}$. The collected sample was kept in a $-2^{\circ} \mathrm{C}$ freezer until it was analyzed. To analyze the terpenes absorbed by the solid absorption tube (KT50601, Supelco, USA) within $48 \mathrm{~h}$, the conditions of an automatic thermal desorption system (TurboMatrix ATD 350, PerkinElmer, USA) were set as shown in (Table 3). In addition, the GC/MSD (GC/MSD-QP2010 Plus, Shimadzu, Japan) for analysis of the desorbed terpenes was set as shown in
(Table 4) to analyze the terpenes.

\section{RESULTS AND DISCUSSION}

Relationship between terpenic compounds contained in Leaves and those that volatilize from the leaves

The essential oils contained in the leaves of the five species of coniferous trees were extracted seasonally and

Table 5. The terpenes content of essential oils from five conifer species

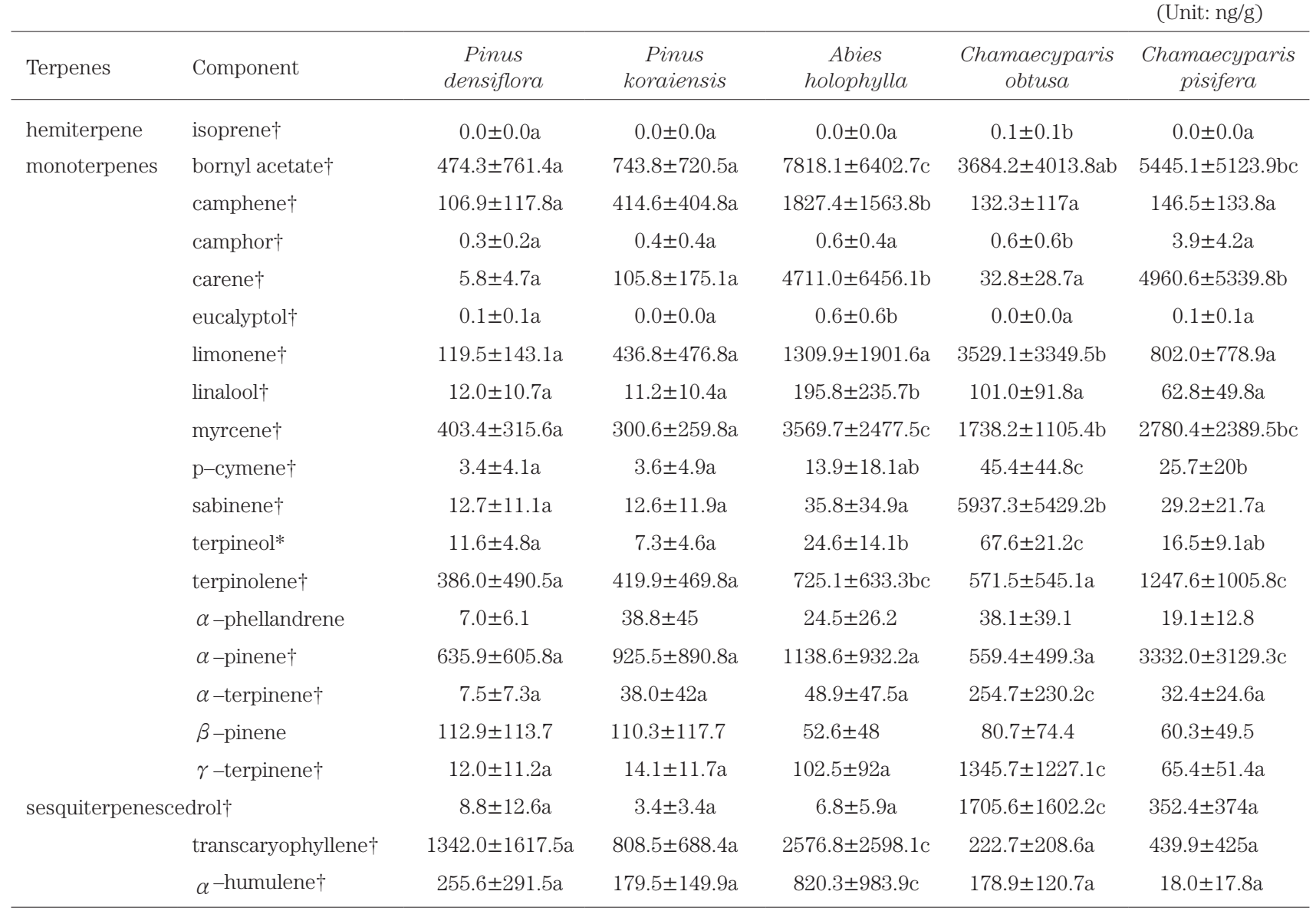

Note: Values are expressed as mean \pm SD $(n=12)$,

Statistical analysis of data was analyzed using one way ANOVA (a, b, c: Duncan's test, *: p $<0.05, \uparrow: p<0.01$ ) 
the concentrations of terpenic compounds were examined. According to the results, of the 21 kinds of terpenic compounds, 19 kinds, except for $\alpha$-phellandrene and $\beta$-pinene, were different in concentrations among the tree species, as shown in (Table 5). The concentrations of terpenic compounds were relatively lower in the essential oils in the leaves of Pinus densiflora and Pinus koraiensis compared to Abies holophylla, Chamaecyparis obtusa, and Chamaecyparis pisifera. On the other hand, the concentrations of eight kinds of terpenic compounds (bornyl acetate, camphene, carene, eucalyptol, linalool, myrcene, trans-caryophyllene, and $\alpha$-humulene) in Abies holophylla leaves, nine kinds of terpenic compounds (isoprene, camphor, limonene, $\mathrm{p}-$ cymene, sabinene, terpineol, $\alpha$-terpinene, $\gamma$-terpinene, and cedrol,) in Chamaecyparis obtuse leaves, and three kinds of terpenic compounds (carene, terpinolene, and $\alpha$-pinene) in Chamaecyparis pisifera leaves were relatively higher compared to other tree species.

However, according to the results of the examination of the concentrations of volatilizing terpenic compounds in the air collected from the airtight container containing coniferous tree leaves, out of 20 kinds of terpenic compounds, the concentrations of 19 kinds, except for terpinolene, were different among the tree species, as shown in (Table 6). Eight kinds of terpenic compounds (eucalyptol, linalool, myrcene, terpineol, $\alpha$-pinene, $\beta$-pinene, and $\gamma$-terpinene) were volatilized relatively more from the leaves of Pinus densiflora among the five coniferous trees species, in addition to 16 kinds (bornyl acetate, camphene, camphor, eucalyptol, limonene, linalool, myrcene, $p$-cymene, terpineol, $\alpha$-phellandrene, $\alpha$ pinene, $\alpha$-terpinene, $\beta$-pinene, cedrol, trans-caryophyllene, and $\alpha$-humulene) from the leaves of Pinus koraiensis, 10 kinds (bornyl acetate, camphene, camphor, eucalyptol, linalool, myrcene, terpineol, cedrol, and $\alpha$-humulene) from the leaves of Abies holophylla, one kind ( $\gamma$-terpinene) from the leaves of Chamaecyparis obtusa, and two kinds (isoprene and cedrol) from the leaves of Chamaecyparis pisifera.

This means that as the emission tendencies of terpenes contained in trees vary with the physical properties, such as molecular weights, water solubility, and volatility of individual materials (Oh et al., 2013), even if the quantity of terpenic compounds in essential oil-contained leaves is large, the quantity of terpenic compounds volatilized is not proportional. Therefore, unless essential oils are to be extracted from the leaves, Chamaecyparis obtusa afforestation is unnecessary to be insisted, but tree species for afforestation should be selected considering various aspects of ecological environments.

\section{Seasonal changes in the concentrations of ter- penic compounds contained in the five species of coniferous trees}

As shown in (Tables 7 and 8), the seasonal concen-

Table 6. The terpenes content from coniferous leaf in sealed container

(Unit: ng/g)

\begin{tabular}{|c|c|c|c|c|c|c|}
\hline Terpenes & Component & $\begin{array}{c}\text { Pinus } \\
\text { densiflora }\end{array}$ & $\begin{array}{c}\text { Pinus } \\
\text { koraiensis }\end{array}$ & $\begin{array}{c}\text { Abies } \\
\text { holophylla }\end{array}$ & $\begin{array}{c}\text { Chamaecyparis } \\
\text { obtusa }\end{array}$ & $\begin{array}{c}\text { Chamaecyparis } \\
\text { pisifera }\end{array}$ \\
\hline isoprene & isoprene† & $246.2 \pm 50.2 b c$ & $210.0 \pm 49.7 \mathrm{~b}$ & $190.8 \pm 78.9 \mathrm{ab}$ & $108.5 \pm 34.1 \mathrm{ac}$ & $316.5 \pm 24.1 \mathrm{c}$ \\
\hline \multirow[t]{16}{*}{ monoterpenes } & bornyl acetate* & $181.5 \pm 83.8 \mathrm{ab}$ & $386.7 \pm 217.2 \mathrm{~b}$ & $295.5 \pm 83.9 b$ & $67.0 \pm 12.2 \mathrm{a}$ & $48.1 \pm 13.9 \mathrm{a}$ \\
\hline & camphene† & $632.5 \pm 292.0 \mathrm{a}$ & $3228.9 \pm 1761.0 \mathrm{~b}$ & $3428.2 \pm 1212.0 \mathrm{~b}$ & $28.7 \pm 0.9 \mathrm{a}$ & $45.7 \pm 11.5 \mathrm{a}$ \\
\hline & camphor $\dagger$ & $13.7 \pm 1.7 \mathrm{bc}$ & $15.8 \pm 3.1 \mathrm{c}$ & $16.3 \pm 3.1 \mathrm{c}$ & $1.7 \pm 0.5 \mathrm{a}$ & $8.9 \pm 5.1 b$ \\
\hline & carenet & $183.0 \pm 37.9 \mathrm{a}$ & $1610.8 \pm 2521.1 \mathrm{a}$ & $4740.7 \pm 496.6 b$ & $20.8 \pm 5.4 .0 \mathrm{a}$ & $1781.7 \pm 145.8 \mathrm{a}$ \\
\hline & eucalyptol $†$ & $8.2 \pm 1.1 \mathrm{c}$ & $9.1 \pm 1.3 \mathrm{c}$ & $9.7 \pm 1.4 \mathrm{c}$ & $0.3 \pm 0.0 \mathrm{a}$ & $4.5 \pm 3.7 \mathrm{~b}$ \\
\hline & limonene* & $301.2 \pm 231.6 \mathrm{a}$ & $2501 \pm 1809.1 b$ & $640.9 \pm 629.1 \mathrm{a}$ & $139.7 \pm 26.1 \mathrm{a}$ & $90.3 \pm 35.0 \mathrm{a}$ \\
\hline & linalool $\dagger$ & $29.5 \pm 2.4 \mathrm{~b}$ & $28.2 \pm 0.4 b$ & $34.3 \pm 7.8 \mathrm{~b}$ & $3.2 \pm 2.0 \mathrm{a}$ & $18.9 \pm 16.0 \mathrm{~b}$ \\
\hline & myrcene† & $19014 \pm 9799.6 b$ & $13813 \pm 7306.5 b$ & $21505.4 \pm 4881.5 b$ & $1290.3 \pm 85.0 \mathrm{a}$ & $1894.7 \pm 832.1 \mathrm{a}$ \\
\hline & p-cymene† & $23.1 \pm 12.8 \mathrm{bc}$ & $31.1 \pm 3.5 \mathrm{c}$ & $13.9 \pm 2.1 \mathrm{ab}$ & $6.6 \pm 1.1 \mathrm{a}$ & $8.9 \pm 3.4 \mathrm{a}$ \\
\hline & terpineol $\dagger$ & $28 \pm 0.5 b$ & $27.9 \pm 0.2 b$ & $27.9 \pm 0.1 b$ & $0.0 \pm 0.0 \mathrm{a}$ & $0.0 \pm 0.0 \mathrm{a}$ \\
\hline & terpinolene & $147.3 \pm 102.7$ & $747.5 \pm 721$ & $201.4 \pm 65.5$ & $25.4 \pm 1.1$ & $70.3 \pm 3.1$ \\
\hline & $\alpha$-phellandrene* & $69.1 \pm 55.5 \mathrm{ab}$ & $146.1 \pm 107.1 \mathrm{~b}$ & $27.5 \pm 5.2 \mathrm{a}$ & $2.0 \pm 0.4 \mathrm{a}$ & $10.7 \pm 7.4 \mathrm{a}$ \\
\hline & $\alpha$-pinene $\dagger$ & $3948.4 \pm 470.1 d$ & $4265.9 \pm 458.8 \mathrm{~d}$ & $2843.2 \pm 876.7 \mathrm{c}$ & $133.5 \pm 19.0 \mathrm{a}$ & $1381.8 \pm 489.1 \mathrm{~b}$ \\
\hline & $\alpha$-terpinene $\dagger$ & $32.8 \pm 17.9 \mathrm{a}$ & $177.1 \pm 103.3 \mathrm{~b}$ & $27.1 \pm 6.5 \mathrm{a}$ & $9.6 \pm 2.4 \mathrm{a}$ & $10.0 \pm 6.3 \mathrm{a}$ \\
\hline & $\beta$-pinene & $2974.7 \pm 1544.9 \mathrm{~b}$ & $1721.5 \pm 851.8 \mathrm{~b}$ & $190.9 \pm 101.5 \mathrm{a}$ & $56.3 \pm 66.0 \mathrm{a}$ & $34.8 \pm 22.7 \mathrm{a}$ \\
\hline & $\gamma$-terpinene $\dagger$ & $34.2 \pm 14.9 \mathrm{ab}$ & $49.8 \pm 13.9 \mathrm{bc}$ & $35.8 \pm 6.8 \mathrm{ab}$ & $59.0 \pm 11.8 \mathrm{c}$ & $15.3 \pm 5.9 \mathrm{a}$ \\
\hline \multirow[t]{3}{*}{ sesquiterpenes } & cedrol $\dagger$ & $28.2 \pm 0.2 b$ & $28.1 \pm 0.0 \mathrm{~b}$ & $28.2 \pm 0.1 b$ & $4.0 \pm 2.3 \mathrm{a}$ & $19.0 \pm 16.2 b$ \\
\hline & transcaryophyllene $\dagger$ & $142.7 \pm 31.5 b c$ & $184.8 \pm 101.1 \mathrm{c}$ & $81.3 \pm 33.1 \mathrm{ab}$ & $4.3 \pm 1.8 \mathrm{a}$ & $16.5 \pm 12.7 \mathrm{a}$ \\
\hline & $\alpha$-humulene $\dagger$ & $34.7 \pm 3.6 \mathrm{~b}$ & $38.6 \pm 10.6 b$ & $33.2 \pm 10.4 \mathrm{~b}$ & $2.6 \pm 1.5 \mathrm{a}$ & $13.8 \pm 11.9 \mathrm{a}$ \\
\hline
\end{tabular}

Note: Values are expressed as mean $\pm \mathrm{SD}(\mathrm{n}=3)$,

Statistical analysis of data was analyzed using one way ANOVA (a, b, c: Duncan's test, *: p<0.05, †: p<0.01) 
Table 7. The seasonal variation of terpenes content from five species essential oils (1)

Pinus densiflora

\begin{tabular}{|c|c|c|c|c|c|c|c|c|}
\hline \multirow{2}{*}{ Component } & \multicolumn{2}{|l|}{ Spring } & \multicolumn{2}{|l|}{ Summer } & \multicolumn{2}{|l|}{ Fall } & \multicolumn{2}{|l|}{ Winter } \\
\hline & Mean \pm SD (ng/g) & $\%$ & Mean \pm SD (ng/g) & $\%$ & Mean \pm SD (ng/g) & $\%$ & Mean \pm SD (ng/g) & $\%$ \\
\hline isoprene & $0.0 \pm 0.0$ & 0.0 & $0.0 \pm 0.0$ & 0.0 & $0.0 \pm 0.0$ & 0.0 & $0.0 \pm 0.0$ & 0.0 \\
\hline bornyl acetate* & $1525.1 \pm 960.8 \mathrm{~b}$ & 16.3 & $270.3 \pm 77.3 \mathrm{a}$ & 7.5 & $46.8 \pm 15.0 \mathrm{a}$ & 3.2 & $54.6 \pm 42.0 \mathrm{a}$ & 4.6 \\
\hline camphene ${ }^{* *}$ & $274.7 \pm 100.8 b$ & 2.9 & $90.7 \pm 74.5 \mathrm{a}$ & 2.5 & $28.5 \pm 16.7 \mathrm{a}$ & 1.9 & $33.6 \pm 20.8 \mathrm{a}$ & 2.8 \\
\hline camphor & $0.1 \pm 0.0$ & 0.0 & $0.2 \pm 0.0$ & 0.0 & $0.3 \pm 0.1$ & 0.0 & $0.3 \pm 0.2$ & 0.0 \\
\hline carene** & $9.2 \pm 2 b$ & 0.1 & $10.7 \pm 2 b$ & 0.3 & $0.6 \pm 0.1 \mathrm{a}$ & 0.0 & $2.4 \pm 0.3 \mathrm{a}$ & 0.2 \\
\hline eucalyptol & $0.0 \pm 0.0$ & 0.0 & $0.0 \pm 0.0$ & 0.0 & $0.0 \pm 0.0$ & 0.0 & $0.0 \pm 0.0$ & 0.0 \\
\hline limonene** & $326.1 \pm 141.6 b$ & 3.5 & $102.7 \pm 25.5 \mathrm{a}$ & 2.8 & $27.0 \pm 14.2 \mathrm{a}$ & 1.8 & $22.2 \pm 11.4 \mathrm{a}$ & 1.9 \\
\hline linalool** & $22.3 \pm 3.5 b$ & 0.2 & $21.0 \pm 6.6 \mathrm{a}$ & 0.6 & $3.8 \pm 1.3 \mathrm{a}$ & 0.3 & $0.9 \pm 0.1 \mathrm{a}$ & 0.1 \\
\hline myrcene** & $7.7 \pm 1.5 \mathrm{a}$ & 0.1 & $285.0 \pm 168.8 \mathrm{a}$ & 7.9 & $750.9 \pm 163 \mathrm{~b}$ & 50.6 & $569.8 \pm 117.8 b$ & 48.0 \\
\hline p-cymene $e^{* *}$ & $1.5 \pm 1.2 \mathrm{a}$ & 0.0 & $9.7 \pm 3 b$ & 0.3 & $0.6 \pm 0.1 \mathrm{a}$ & 0.0 & $1.6 \pm 0.2 \mathrm{a}$ & 0.1 \\
\hline sabinene** & $27.6 \pm 1.3 \mathrm{c}$ & 0.3 & $16.6 \pm 6.6 \mathrm{~b}$ & 0.5 & $3.8 \pm 1.0 \mathrm{a}$ & 0.3 & $2.7 \pm 1.2 \mathrm{a}$ & 0.2 \\
\hline terpineol** & $10.4 \pm 0.2 b$ & 0.1 & $14.8 \pm 4.4 \mathrm{ab}$ & 0.4 & $15.5 \pm 2.1 b$ & 1.0 & $5.3 \pm 1.5 \mathrm{a}$ & 0.4 \\
\hline terpinolene ${ }^{* *}$ & $1086.9 \pm 521.7 \mathrm{~b}$ & 11.6 & $317.9 \pm 43.0 \mathrm{a}$ & 8.8 & $82.9 \pm 56.5 \mathrm{a}$ & 5.6 & $56.0 \pm 25.1 \mathrm{a}$ & 4.7 \\
\hline$\alpha$-phellandrene ${ }^{* *}$ & $8.1 \pm 1.7 \mathrm{~b}$ & 0.1 & $15.0 \pm 5.6 \mathrm{~b}$ & 0.4 & $2.0 \pm 0.2 \mathrm{a}$ & 0.1 & $2.6 \pm 0.4 a b$ & 0.2 \\
\hline$\alpha$-pinene ${ }^{* *}$ & $1552.3 \pm 131.8 \mathrm{~b}$ & 16.5 & $586.5 \pm 397.2 \mathrm{a}$ & 16.2 & $195.8 \pm 86.5 \mathrm{a}$ & 13.2 & $208.9 \pm 88.1 \mathrm{a}$ & 17.6 \\
\hline$\alpha$-terpinene ${ }^{* *}$ & $11.4 \pm 0.5 b$ & 0.1 & $16.0 \pm 6.3 \mathrm{~b}$ & 0.4 & $1.7 \pm 0.1 \mathrm{a}$ & 0.1 & $0.8 \pm 0.4 \mathrm{a}$ & 0.1 \\
\hline$\beta$-pinene $* *$ & $284.7 \pm 38.6 \mathrm{c}$ & 3.0 & $109.5 \pm 61.2 \mathrm{~b}$ & 3.0 & $28.8 \pm 10.5 a$ & 1.9 & $28.5 \pm 13.1 \mathrm{a}$ & 2.4 \\
\hline$\gamma$-terpinene ${ }^{* *}$ & $22.6 \pm 2.1 b$ & 0.2 & $21.3 \pm 8.4 b$ & 0.6 & $3.0 \pm 0.2 \mathrm{a}$ & 0.2 & $0.8 \pm 0.3 \mathrm{a}$ & 0.1 \\
\hline cedrol** & $27.2 \pm 12.9 \mathrm{~b}$ & 0.3 & $5.6 \pm 2.1 \mathrm{a}$ & 0.2 & $1.6 \pm 2.1 \mathrm{a}$ & 0.1 & $0.8 \pm 0.8 \mathrm{a}$ & 0.1 \\
\hline trans-caryophyllene** & $3532.0 \pm 1800.6 \mathrm{~b}$ & 37.7 & $1431.1 \pm 184.9 \mathrm{a}$ & 39.6 & $240.2 \pm 72.3 \mathrm{a}$ & 16.2 & $164.5 \pm 27.9 \mathrm{a}$ & 13.9 \\
\hline$\alpha$-humulene ${ }^{* *}$ & $650.1 \pm 304.3 \mathrm{~b}$ & 6.9 & $290.7 \pm 17.1 \mathrm{a}$ & 8.0 & $50.7 \pm 13.9 \mathrm{a}$ & 3.4 & $30.9 \pm 8.0 \mathrm{a}$ & 2.6 \\
\hline total & 9380.0 & 100.0 & 3615.3 & 100.0 & 1484.5 & 100.0 & 1187.2 & 100.0 \\
\hline
\end{tabular}

\section{Pinus koraiensis}

\begin{tabular}{|c|c|c|c|c|c|c|c|c|}
\hline \multirow{2}{*}{ Component } & \multicolumn{2}{|l|}{ Spring } & \multicolumn{2}{|l|}{ Summer } & \multicolumn{2}{|l|}{ Fall } & \multicolumn{2}{|l|}{ Winter } \\
\hline & Mean士SD (ng/g) & $\%$ & Mean \pm SD (ng/g) & $\%$ & Mean \pm SD (ng/g) & $\%$ & Mean \pm SD (ng/g) & $\%$ \\
\hline isoprene & $0.0 \pm 0.0$ & 0.0 & $0.0 \pm 0.0$ & 0.0 & $0.0 \pm 0.0$ & 0.0 & $0.0 \pm 0.0$ & 0.0 \\
\hline bornyl acetate* & $1489.4 \pm 760.0 \mathrm{~b}$ & 17.7 & $1119.7 \pm 515.3 \mathrm{~b}$ & 17.2 & $166.7 \pm 61.2 \mathrm{a}$ & 9.4 & $199.3 \pm 134.2 \mathrm{a}$ & 12.4 \\
\hline camphene & $844.9 \pm 456.2$ & 10.1 & $533.8 \pm 401.9$ & 8.2 & $152.3 \pm 61.0$ & 8.6 & $127.4 \pm 43.3$ & 7.9 \\
\hline camphor & $0.1 \pm 0.0$ & 0.0 & $0.3 \pm 0.1$ & 0.0 & $0.3 \pm 0.1$ & 0.0 & $0.8 \pm 0.6$ & 0.0 \\
\hline carene & $87.9 \pm 77.8$ & 1.0 & $214.1 \pm 346.2$ & 3.3 & $69.9 \pm 119.4$ & 3.9 & $51.1 \pm 62.5$ & 3.2 \\
\hline eucalyptol & $0.0 \pm 0.0$ & 0.0 & $0.0 \pm 0.0$ & 0.0 & $0.0 \pm 0.0$ & 0.0 & $0.0 \pm 0.0$ & 0.0 \\
\hline limonene & $898.0 \pm 592.4$ & 10.7 & $643.8 \pm 419.5$ & 9.9 & $110.9 \pm 48.1$ & 6.2 & $94.5 \pm 51.5$ & 5.9 \\
\hline linalool** & $14.0 \pm 6.6 \mathrm{ab}$ & 0.2 & $24.1 \pm 9.6 \mathrm{~b}$ & 0.4 & $3.9 \pm 0.7 \mathrm{a}$ & 0.2 & $2.6 \pm 1.7 \mathrm{a}$ & 0.2 \\
\hline myrcene & $57.9 \pm 50.4$ & 0.7 & $190.4 \pm 60.1$ & 2.9 & $499.1 \pm 283.4$ & 28.0 & $454.7 \pm 288.7$ & 28.3 \\
\hline p-cymene ${ }^{* *}$ & $1.1 \pm 0.9 \mathrm{a}$ & 0.0 & $11.1 \pm 3.4 \mathrm{~b}$ & 0.2 & $0.8 \pm 0.1 \mathrm{a}$ & 0.0 & $1.2 \pm 0.4 \mathrm{a}$ & 0.1 \\
\hline sabinene & $21.4 \pm 17.0$ & 0.3 & $21.1 \pm 4.4$ & 0.3 & $4.3 \pm 2.1$ & 0.2 & $3.3 \pm 2.8$ & 0.2 \\
\hline terpineol & $5.5 \pm 4.0$ & 0.1 & $8.6 \pm 7.9$ & 0.1 & $8.2 \pm 3.9$ & 0.5 & $6.7 \pm 3.4$ & 0.4 \\
\hline terpinolene & $783.8 \pm 575.8$ & 9.3 & $679.4 \pm 528.9$ & 10.4 & $124.5 \pm 86.1$ & 7.0 & $91.8 \pm 52.6$ & 5.7 \\
\hline$\alpha$-phellandrene & $75.6 \pm 66.8$ & 0.9 & $58.5 \pm 39.3$ & 0.9 & $10.5 \pm 6.4$ & 0.6 & $10.5 \pm 8.4$ & 0.7 \\
\hline$\alpha$-pinene* & $1914.3 \pm 1015.8 \mathrm{~b}$ & 22.8 & $1157.0 \pm 802.4 \mathrm{ab}$ & 17.8 & $345.8 \pm 86.6 \mathrm{a}$ & 19.4 & $284.9 \pm 119.6 \mathrm{a}$ & 17.8 \\
\hline$\alpha$-terpinene & $73.4 \pm 60.1$ & 0.9 & $58.1 \pm 33.9$ & 0.9 & $10.5 \pm 5.8$ & 0.6 & $9.7 \pm 8.6$ & 0.6 \\
\hline$\beta$-pinene & $229.2 \pm 157.9$ & 2.7 & $148.3 \pm 93.9$ & 2.3 & $33.8 \pm 10.1$ & 1.9 & $29.9 \pm 19.0$ & 1.9 \\
\hline$\gamma$-terpinene ${ }^{* *}$ & $21.6 \pm 4.6 \mathrm{~b}$ & 0.3 & $27.7 \pm 4.9 \mathrm{~b}$ & 0.4 & $4.4 \pm 0.8 \mathrm{a}$ & 0.2 & $2.5 \pm 1.0 \mathrm{a}$ & 0.2 \\
\hline cedrol & $7.5 \pm 4.1$ & 0.1 & $3.9 \pm 0.7$ & 0.1 & $0.2 \pm 0.1$ & 0.0 & $1.7 \pm 1.1$ & 0.1 \\
\hline trans-caryophyllene ${ }^{* *}$ & $1547.6 \pm 389.8 b$ & 18.4 & $1306.8 \pm 331.8 b$ & 20.1 & $189.5 \pm 17.5 \mathrm{a}$ & 10.6 & $189.8 \pm 52 \mathrm{a}$ & 11.8 \\
\hline$\alpha$-humulene ${ }^{* *}$ & $330.5 \pm 84.6 \mathrm{~b}$ & 3.9 & $299.8 \pm 70.7 \mathrm{~b}$ & 4.6 & $45.5 \pm 4.3 \mathrm{a}$ & 2.6 & $41.9 \pm 10.5 \mathrm{a}$ & 2.6 \\
\hline total & 8403.7 & 100.0 & 6506.5 & 100.0 & 1781.1 & 100.0 & 1604.3 & 100.0 \\
\hline
\end{tabular}


Abies holophylla

\begin{tabular}{|c|c|c|c|c|c|c|c|c|}
\hline \multirow{2}{*}{ Component } & \multicolumn{2}{|l|}{ Spring } & \multicolumn{2}{|l|}{ Summer } & \multicolumn{2}{|l|}{ Fall } & \multicolumn{2}{|l|}{ Winter } \\
\hline & Mean \pm SD (ng/g) & $\%$ & Mean \pm SD (ng/g) & $\%$ & Mean \pm SD (ng/g) & $\%$ & Mean士SD (ng/g) & $\%$ \\
\hline isoprene & $0.0 \pm 0.0$ & 0.0 & $0.0 \pm 0.0$ & 0.0 & $0.0 \pm 0.0$ & 0.0 & $0.0 \pm 0.0$ & 0.0 \\
\hline bornyl acetate ${ }^{* *}$ & $14433.3 \pm 2744.9 b$ & 46.9 & $13140.8 \pm 1578.2 b$ & 27.7 & $1849.8 \pm 537.5 \mathrm{a}$ & 15.7 & $1848.4 \pm 352.8 \mathrm{a}$ & 18.5 \\
\hline camphene ${ }^{* *}$ & $3390.9 \pm 1380.2 b$ & 11.0 & $2905.9 \pm 940 b$ & 6.1 & $510.4 \pm 78.3 a$ & 4.3 & $502.3 \pm 90.2 \mathrm{a}$ & 5.0 \\
\hline camphor** & $0.1 \pm 0.0 \mathrm{~b}$ & 0.0 & $0.6 \pm 0.2 \mathrm{a}$ & 0.0 & $0.5 \pm 0.1 \mathrm{a}$ & 0.0 & $1.0 \pm 0.2 \mathrm{c}$ & 0.0 \\
\hline carene** & $25.3 \pm 10.2 \mathrm{a}$ & 0.1 & $14652.2 \pm 5201.3 \mathrm{~b}$ & 30.9 & $2198.5 \pm 163.4 \mathrm{a}$ & 18.6 & $1967.8 \pm 481.5 \mathrm{a}$ & 19.7 \\
\hline eucalyptol* & $0.0 \pm 0.0 \mathrm{a}$ & 0.0 & $0.8 \pm 0.3 \mathrm{ab}$ & 0.0 & $1.1 \pm 0.8 \mathrm{~b}$ & 0.0 & $0.3 \pm 0.1 \mathrm{a}$ & 0.0 \\
\hline limonene & $2413.8 \pm 2404.7$ & 7.8 & $2304.0 \pm 2719.7$ & 4.9 & $240.2 \pm 106.3$ & 2.0 & $281.6 \pm 289.1$ & 2.8 \\
\hline linalool & $286.2 \pm 294.1$ & 0.9 & $423.6 \pm 224.9$ & 0.9 & $42.5 \pm 17.9$ & 0.4 & $30.7 \pm 41.7$ & 0.3 \\
\hline myrcene ${ }^{* *}$ & $20.2 \pm 7.4 \mathrm{a}$ & 0.1 & $4321.2 \pm 1976.7 \mathrm{~b}$ & 9.1 & $5797.4 \pm 756.9 \mathrm{~b}$ & 49.2 & $4139.9 \pm 1264.6 \mathrm{~b}$ & 41.4 \\
\hline p-cymene ${ }^{* *}$ & $4.7 \pm 1.1 \mathrm{~b}$ & 0.0 & $43.4 \pm 3.4 \mathrm{a}$ & 0.1 & $3.9 \pm 3.7 \mathrm{a}$ & 0.0 & $3.2 \pm 0.2 \mathrm{a}$ & 0.0 \\
\hline sabinene** & $44.6 \pm 16.9 \mathrm{~b}$ & 0.1 & $82.7 \pm 24.8 c$ & 0.2 & $10.4 \pm 2.5 a$ & 0.1 & $5.5 \pm 1.9 \mathrm{a}$ & 0.1 \\
\hline terpineol & $19.8 \pm 18.4$ & 0.1 & $38.1 \pm 8.9$ & 0.1 & $26.2 \pm 12.2$ & 0.2 & $14.1 \pm 6.8$ & 0.1 \\
\hline terpinolene ${ }^{* *}$ & $1298.3 \pm 446.8 b$ & 4.2 & $1272.4 \pm 346.4 \mathrm{~b}$ & 2.7 & $175.3 \pm 28.6 \mathrm{a}$ & 1.5 & $154.4 \pm 35.0 \mathrm{a}$ & 1.5 \\
\hline$\alpha$-phellandrene ${ }^{* *}$ & $22.0 \pm 8.8 \mathrm{a}$ & 0.1 & $64.3 \pm 16.3 b$ & 0.1 & $5.9 \pm 0.7 \mathrm{a}$ & 0.1 & $5.7 \pm 0.6 \mathrm{a}$ & 0.1 \\
\hline$\alpha$-pinene ${ }^{* *}$ & $1973.3 \pm 561.2 \mathrm{~b}$ & 6.4 & $1931.5 \pm 691.7 \mathrm{~b}$ & 4.1 & $341.7 \pm 79.4 \mathrm{a}$ & 2.9 & $307.5 \pm 48.0 \mathrm{a}$ & 3.1 \\
\hline$\alpha$-terpinene ${ }^{* *}$ & $60.2 \pm 25.2 \mathrm{~b}$ & 0.2 & $113.7 \pm 29.6 c$ & 0.2 & $12.8 \pm 2.3 \mathrm{a}$ & 0.1 & $8.7 \pm 2.5 \mathrm{a}$ & 0.1 \\
\hline$\beta$-pinene ${ }^{* *}$ & $81.2 \pm 20.1 b$ & 0.3 & $102.5 \pm 50.7 \mathrm{~b}$ & 0.2 & $15.5 \pm 4.7 \mathrm{a}$ & 0.1 & $11.0 \pm 2.5 \mathrm{a}$ & 0.1 \\
\hline$\gamma$-terpinene ${ }^{* *}$ & $161.9 \pm 60.9 \mathrm{~b}$ & 0.5 & $203.3 \pm 54.1 b$ & 0.4 & $24.6 \pm 3.6 \mathrm{a}$ & 0.2 & $20.0 \pm 4.8 \mathrm{a}$ & 0.2 \\
\hline cedrol* & $11.2 \pm 5.8 \mathrm{~b}$ & 0.0 & $11.1 \pm 3.8 \mathrm{~b}$ & 0.0 & $0.3 \pm 0.2 \mathrm{a}$ & 0.0 & $4.3 \pm 3.4 \mathrm{ab}$ & 0.0 \\
\hline trans-caryophyllene* & $5002.3 \pm 2257 \mathrm{~b}$ & 16.2 & $4377.2 \pm 2213.1 b$ & 9.2 & $405.3 \pm 58 \mathrm{a}$ & 3.4 & $522.2 \pm 196.9 \mathrm{a}$ & 5.2 \\
\hline$\alpha$-humulene & $1541.9 \pm 1135.3$ & 5.0 & $1439.1 \pm 1146.4$ & 3.0 & $128.1 \pm 16.1$ & 1.1 & $171.7 \pm 124.6$ & 1.7 \\
\hline total & 30791.2 & 100.0 & 47428.4 & 100.0 & 11790.4 & 100.0 & 10000.3 & 100.0 \\
\hline
\end{tabular}

Note: Values are expressed as mean \pm SD $(n=3)$,

Statistical analysis of data was analyzed using one way ANOVA (a, b, c: Duncan's test, *: p<0.05, †: p<0.01)

Table 8. The seasonal variation of terpenes content from five species essential oils (2)

Chamaecyparis obtusa

\begin{tabular}{|c|c|c|c|c|c|c|c|c|}
\hline \multirow{2}{*}{ Component } & \multicolumn{2}{|l|}{ Spring } & \multicolumn{2}{|l|}{ Summer } & \multicolumn{2}{|l|}{ Fall } & \multicolumn{2}{|l|}{ Winter } \\
\hline & Mean $\pm S D$ (ng/g) & $\%$ & Mean $\pm S D(n g / g)$ & $\%$ & Mean \pm SD (ng/g) & $\%$ & Mean士SD (ng/g) & $\%$ \\
\hline isoprene $\dagger$ & $0.0 \pm 0.0 \mathrm{a}$ & 0.0 & $0.1 \pm 0.1 \mathrm{ab}$ & 0.0 & $0.2 \pm 0.1 b$ & 0.0 & $0.0 \pm 0.0 \mathrm{a}$ & 0.0 \\
\hline bornyl acetate & $7140.9 \pm 5967.6$ & 19.1 & $5944.0 \pm 1650.2$ & 19.4 & $777.0 \pm 253.5$ & 12.2 & $874.5 \pm 322$ & 13.6 \\
\hline camphene† & $235.7 \pm 77.9 \mathrm{~b}$ & 0.6 & $235.3 \pm 70.3 \mathrm{~b}$ & 0.8 & $28.5 \pm 7.1 \mathrm{a}$ & 0.4 & $29.4 \pm 9.5 \mathrm{a}$ & 0.5 \\
\hline camphor* & $0.4 \pm 0.1 \mathrm{a}$ & 0.0 & $0.4 \pm 0.1 \mathrm{a}$ & 0.0 & $0.3 \pm 0.1 \mathrm{a}$ & 0.0 & $1.3 \pm 0.7 \mathrm{~b}$ & 0.0 \\
\hline carenet & $44.9 \pm 12.7 \mathrm{~b}$ & 0.1 & $68.7 \pm 19.6 c$ & 0.2 & $5.4 \pm 4.5 \mathrm{a}$ & 0.1 & $12.0 \pm 4.2 \mathrm{a}$ & 0.2 \\
\hline eucalyptol & $0.0 \pm 0.0$ & 0.0 & $0.0 \pm 0.0$ & 0.0 & $0.0 \pm 0.0$ & 0.0 & $0.0 \pm 0.0$ & 0.0 \\
\hline limonene $\dagger$ & $7805.8 \pm 1967.3 \mathrm{c}$ & 20.9 & $5030.2 \pm 1373.4 b$ & 16.4 & $745.4 \pm 166.5 \mathrm{a}$ & 11.7 & $534.6 \pm 171.9 \mathrm{a}$ & 8.3 \\
\hline linalool $\dagger$ & $190.5 \pm 57.6 \mathrm{~b}$ & 0.5 & $175.4 \pm 48.3 \mathrm{~b}$ & 0.6 & $23.2 \pm 3.1 \mathrm{a}$ & 0.4 & $14.7 \pm 5.0 \mathrm{a}$ & 0.2 \\
\hline myrcene $\dagger$ & $36.2 \pm 9.9 \mathrm{a}$ & 0.1 & $1917.6 \pm 335 b$ & 6.2 & $2503.2 \pm 383.2 \mathrm{~b}$ & 39.2 & $2495.4 \pm 573.8 b$ & 38.7 \\
\hline p-cymene $\dagger$ & $63.4 \pm 28.6 b$ & 0.2 & $100.1 \pm 35.2 b$ & 0.3 & $7.2 \pm 1.1 \mathrm{a}$ & 0.1 & $10.7 \pm 2.7 \mathrm{a}$ & 0.2 \\
\hline sabinene $\dagger$ & $11796.5 \pm 3589.4 \mathrm{~b}$ & 31.6 & $9571.2 \pm 3163.8 \mathrm{~b}$ & 31.2 & $1191.4 \pm 344.8 \mathrm{a}$ & 18.7 & $1189.9 \pm 458.0 \mathrm{a}$ & 18.5 \\
\hline terpineol & $88.9 \pm 23.3$ & 0.2 & $61.5 \pm 19.3$ & 0.2 & $68.2 \pm 13.6$ & 1.1 & $51.6 \pm 15.3$ & 0.8 \\
\hline terpinolene $\dagger$ & $1265.1 \pm 308.2 \mathrm{c}$ & 3.4 & $822.5 \pm 226.1 b$ & 2.7 & $112.3 \pm 27.2 \mathrm{a}$ & 1.8 & $85.7 \pm 29.4 \mathrm{a}$ & 1.3 \\
\hline$\alpha$-phellandrene ${ }^{\dagger}$ & $39.1 \pm 11.1 b$ & 0.1 & $96.0 \pm 24.6 \mathrm{c}$ & 0.3 & $8.5 \pm 1.3 \mathrm{a}$ & 0.1 & $8.6 \pm 2.1 \mathrm{a}$ & 0.1 \\
\hline$\alpha$-pinene ${ }^{\dagger}$ & $1006.8 \pm 341.2 \mathrm{~b}$ & 2.7 & $988.2 \pm 315.8 b$ & 3.2 & $120.9 \pm 29.3 \mathrm{a}$ & 1.9 & $121.5 \pm 39.9 \mathrm{a}$ & 1.9 \\
\hline$\alpha$-terpinene ${ }^{\dagger}$ & $496.0 \pm 117.4 \mathrm{~b}$ & 1.3 & $429.4 \pm 118.8 b$ & 1.4 & $48.3 \pm 11.0 \mathrm{a}$ & 0.8 & $44.8 \pm 16.4 \mathrm{a}$ & 0.7 \\
\hline$\beta$-pinene ${ }^{\dagger}$ & $134.5 \pm 43.2 \mathrm{~b}$ & 0.4 & $158.7 \pm 45.4 \mathrm{~b}$ & 0.5 & $15.6 \pm 3.3 \mathrm{a}$ & 0.2 & $14 \pm 4.9 \mathrm{a}$ & 0.2 \\
\hline$\gamma$-terpinene ${ }^{\dagger}$ & $2811.6 \pm 645 b$ & 7.5 & $2062.8 \pm 534.6 \mathrm{~b}$ & 6.7 & $253.1 \pm 59.7 \mathrm{a}$ & 4.0 & $255.1 \pm 83.1 \mathrm{a}$ & 4.0 \\
\hline cedrol $\dagger$ & $3587.1 \pm 1209.7 \mathrm{~b}$ & 9.6 & $2470.2 \pm 1076.6 \mathrm{~b}$ & 8.0 & $368.4 \pm 151.4 \mathrm{a}$ & 5.8 & $396.5 \pm 181.6 \mathrm{a}$ & 6.2 \\
\hline
\end{tabular}




\begin{tabular}{lcrcrcrrr} 
trans-caryophyllene $\dagger$ & $473.1 \pm 193 \mathrm{c}$ & 1.3 & $297.5 \pm 136.9 \mathrm{bc}$ & 1.0 & $73.6 \pm 22.4 \mathrm{~b}$ & 1.2 & $46.7 \pm 22.1 \mathrm{a}$ & 0.7 \\
$\alpha$-humulene* & $145.9 \pm 75.5 \mathrm{a}$ & 0.4 & $277.4 \pm 26.1 \mathrm{ab}$ & 0.9 & $32.4 \pm 5.6 \mathrm{~b}$ & 0.5 & $259.9 \pm 124.4 \mathrm{~b}$ & 4.0 \\
total & 37362.4 & 100.0 & 30707.2 & 100.0 & 6383.1 & 100.0 & 6446.9 & 100.0 \\
\hline
\end{tabular}

\section{Chamaecyparis pisifera}

\begin{tabular}{|c|c|c|c|c|c|c|c|c|}
\hline \multirow{2}{*}{ Component } & \multicolumn{2}{|l|}{ Spring } & \multicolumn{2}{|l|}{ Summer } & \multicolumn{2}{|l|}{ Fall } & \multicolumn{2}{|l|}{ Winter } \\
\hline & Mean士SD (ng/g) & $\%$ & Mean $\pm S D(n g / g)$ & $\%$ & Mean士SD (ng/g) & $\%$ & Mean \pm SD (ng/g) & $\%$ \\
\hline isoprene & $0.0 \pm 0.0$ & 0.0 & $0.0 \pm 0.0$ & 0.0 & $0.0 \pm 0.0$ & 0.0 & $0.0 \pm 0.0$ & 0.0 \\
\hline bornyl acetate $\dagger$ & $12696.7 \pm 3039.5 c$ & 45.5 & $6339.6 \pm 2225.3 b$ & 24.4 & $1167.6 \pm 281.8 \mathrm{a}$ & 11.7 & $1576.5 \pm 393.1 \mathrm{a}$ & 10.2 \\
\hline camphenet & $342.6 \pm 101.3 \mathrm{~b}$ & 1.2 & $120.3 \pm 76.8 \mathrm{a}$ & 0.5 & $40.2 \pm 11.2 \mathrm{a}$ & 0.4 & $82.5 \pm 18.1 \mathrm{a}$ & 0.5 \\
\hline camphor & $3.0 \pm 3.7$ & 0.0 & $2.4 \pm 2.9$ & 0.0 & $5.1 \pm 4.5$ & 0.1 & $5.0 \pm 6.7$ & 0.0 \\
\hline carene* & $27.8 \pm 1.4 \mathrm{a}$ & 0.1 & $11857.3 \pm 6502.7 \mathrm{~b}$ & 45.6 & $3170.1 \pm 987 a$ & 31.8 & $4786.8 \pm 928.4 a$ & 30.9 \\
\hline eucalyptol $\dagger$ & $0.1 \pm 0.0 \mathrm{a}$ & 0.0 & $0.0 \pm 0.0 \mathrm{a}$ & 0.0 & $0.1 \pm 0.1 b$ & 0.0 & $0.1 \pm 0 \mathrm{~b}$ & 0.0 \\
\hline limonene† & $1951.8 \pm 570.4 \mathrm{~b}$ & 7.0 & $778.3 \pm 265.5 \mathrm{a}$ & 3.0 & $215.7 \pm 34.5 \mathrm{a}$ & 2.2 & $262.1 \pm 51.7 \mathrm{a}$ & 1.7 \\
\hline linalool $†$ & $120.3 \pm 47.8 \mathrm{c}$ & 0.4 & $83.0 \pm 33.5 b c$ & 0.3 & $18.9 \pm 6.2 \mathrm{a}$ & 0.2 & $29.0 \pm 3.2 \mathrm{ab}$ & 0.2 \\
\hline myrcene† & $21.9 \pm 0.7 \mathrm{a}$ & 0.1 & $1438.4 \pm 560.1 b$ & 5.5 & $3737.0 \pm 176 c$ & 37.5 & $5923.9 \pm 864.6 \mathrm{~d}$ & 38.3 \\
\hline p-cymene† & $48.5 \pm 4.2 \mathrm{~b}$ & 0.2 & $38.2 \pm 12.4 \mathrm{~b}$ & 0.1 & $5.3 \pm 0.5 \mathrm{a}$ & 0.1 & $10.5 \pm 3.9 \mathrm{a}$ & 0.1 \\
\hline sabinene $\dagger$ & $56.6 \pm 11.6 \mathrm{a}$ & 0.2 & $38.0 \pm 13.5 b$ & 0.1 & $9.4 \pm 1.5 \mathrm{a}$ & 0.1 & $12.4 \pm 3.3 \mathrm{a}$ & 0.1 \\
\hline terpineol* & $12.6 \pm 1.9 \mathrm{a}$ & 0.0 & $8.6 \pm 1.9 \mathrm{a}$ & 0.0 & $16.3 \pm 4.1 \mathrm{a}$ & 0.2 & $28.4 \pm 10.2 c$ & 0.2 \\
\hline terpinolene ${ }^{\dagger}$ & $2654.5 \pm 147.8 \mathrm{a}$ & 9.5 & $1484.1 \pm 657.8 b$ & 5.7 & $358.5 \pm 80.5 c$ & 3.6 & $493.2 \pm 98.4 \mathrm{a}$ & 3.2 \\
\hline$\alpha$-phellandrene ${ }^{\dagger}$ & $24.2 \pm 1.2 \mathrm{~b}$ & 0.1 & $34.9 \pm 11 b$ & 0.1 & $6.3 \pm 1.8 \mathrm{a}$ & 0.1 & $10.9 \pm 2.2 \mathrm{a}$ & 0.1 \\
\hline$\alpha$-pinene pi $^{\dagger}$ & $7917.1 \pm 2665.4 b$ & 28.3 & $2546.6 \pm 1585 a$ & 9.8 & $953.0 \pm 243 a$ & 9.6 & $1911.2 \pm 453.1 \mathrm{a}$ & 12.3 \\
\hline$\alpha$-terpinene ${ }^{\dagger}$ & $60.7 \pm 6.6 \mathrm{~b}$ & 0.2 & $46.9 \pm 19.3 b$ & 0.2 & $10.0 \pm 1.3 \mathrm{a}$ & 0.1 & $11.9 \pm 1.5 \mathrm{a}$ & 0.1 \\
\hline$\beta$-pinene ${ }^{\dagger}$ & $130.6 \pm 24.8 \mathrm{c}$ & 0.5 & $64.3 \pm 33.7 \mathrm{~b}$ & 0.2 & $16.9 \pm 3.9 \mathrm{ab}$ & 0.2 & $29.4 \pm 5 a$ & 0.2 \\
\hline$\gamma$-terpinene ${ }^{\dagger}$ & $132.4 \pm 9.5 \mathrm{c}$ & 0.5 & $87.2 \pm 31.1 b$ & 0.3 & $19.2 \pm 3.1 \mathrm{a}$ & 0.2 & $22.5 \pm 3.2 \mathrm{a}$ & 0.1 \\
\hline cedrol & $743.9 \pm 442.2$ & 2.7 & $435.9 \pm 380.7$ & 1.7 & $88.3 \pm 73.9$ & 0.9 & $141.4 \pm 107.2$ & 0.9 \\
\hline trans-caryophyllene* & $964.6 \pm 442.3 b$ & 3.5 & $529.9 \pm 275.7 \mathrm{ab}$ & 2.0 & $129.3 \pm 59.1 \mathrm{a}$ & 1.3 & $135.6 \pm 97.1 \mathrm{a}$ & 0.9 \\
\hline$\alpha$-humulene ${ }^{\dagger}$ & $16.7 \pm 12.7 \mathrm{a}$ & 0.1 & $43.7 \pm 10.5 b$ & 0.2 & $6.7 \pm 0.5 \mathrm{a}$ & 0.1 & $4.8 \pm 3.1 \mathrm{a}$ & 0.0 \\
\hline total & 27926.6 & 100.0 & 25977.6 & 100.0 & 9973.9 & 100.0 & 15478.1 & 100.0 \\
\hline
\end{tabular}

Note: Values are expressed as mean \pm SD $(n=3)$,

Statistical analysis of data was analyzed using one way ANOVA (a, b, c: Duncan's test, *: p<0.05, †: p<0.01)

trations of all the 21 kinds of terpenic compounds were the highest in summer in the case of Abies holophylla and in spring in the cases of Pinus densiflora, Pinus koraiensis, Chamaecyparis obtusa, and Chamaecyparis pisifera according to analyses. In addition, seasonal differences in the concentrations of terpenic compounds were also found among the kinds of terpenic compounds. Eighteen kinds of terpenic compounds in the case of Pinus densiflora, seven kinds in the case of Pinus koraiensis, 16 kinds in the case of Abies holophylla, 18 kinds in the case of Chamaecyparis obtusa, and 18 kinds in the case of Chamaecyparis pisifera showed significant seasonal differences in concentrations in the analysis. The concentrations of most of the various terpenic compounds contained in the leaves of the five species of coniferous trees were higher in spring and summer, except for the concentrations of myrcene, which showed a tendency to increase in autumn and winter.

The reason why the concentrations of terpenic compounds contained in coniferous trees are higher in summer or spring as such is judged to be that germination, growth, and development are the most active in spring
(Kim, 2001) and that the time of biosynthesis of terpenic compounds is seasons in which physiological activities are vigorous (Song, 1995). The concentration of myrcene was shown to commonly increase as seasons changed from spring to summer, autumn, and winter in order of precedence in the essential oils extracted from the leaves of the five species of coniferous trees. This was consistent with the result of a previous study conducted by Son and Hwang (Son and Hwang, 1990), indicating that the concentration of myrcene contained in the leaves of Pinus densiflora, Pinus rigida Mill., and Pinus thunbergii Parl. increased until July, but not with the result indicating that the concentration decreased in October.

The correlations among various terpenic compounds that increased or decreased seasonally were analyzed and the results are as shown in (Tables 9 and 10). That is, the concentrations of terpenic compounds contained in the essential oils extracted from the leaves of five species of coniferous trees generally showed positive $(+)$ correlations with each other, except for several terpenic compounds that showed negative $(-)$ correlations with each other. In the essential oil extracted from the leaves of Pinus densiflora, myrcene showed negative (-) cor- 
Table 9. The correlation among the terpenes (1)

\begin{tabular}{|c|c|c|c|c|c|c|c|c|c|c|c|c|}
\hline & & isoprene & $\begin{array}{c}\text { bornyl } \\
\text { acetate }\end{array}$ & camphene & camphor & carene & eucalyptol & limonene & linalool & myrcene & p-cymene & sabinene \\
\hline \multirow[t]{5}{*}{ isoprene } & P. densiflora & 1 & & & & & & & & & & \\
\hline & P. koraiensis & 1 & & & & & & & & & & \\
\hline & A .holophylla & 1 & & & & & & & & & & \\
\hline & C. obtusa & 1 & & & & & & & & & & \\
\hline & C. pisifera & 1 & & & & & & & & & & \\
\hline bornyl & P. densiflora & ND & & & & & & & & & & \\
\hline \multirow[t]{4}{*}{ acetate } & P. koraiensis & $\mathrm{ND}$ & 1 & & & & & & & & & \\
\hline & A .holophylla & $\mathrm{ND}$ & 1 & & & & & & & & & \\
\hline & C. obtusa & -0.242 & 1 & & & & & & & & & \\
\hline & C. pisifera & $\mathrm{ND}$ & 1 & & & & & & & & & \\
\hline \multirow[t]{5}{*}{ camphene } & P. densiflora & ND & $0.925 \dagger$ & 1 & & & & & & & & \\
\hline & P. koraiensis & ND & $0.839 \dagger$ & 1 & & & & & & & & \\
\hline & A .holophylla & $\mathrm{ND}$ & $0.957 \dagger$ & 1 & & & & & & & & \\
\hline & C. obtusa & -0.239 & $0.898 \dagger$ & 1 & & & & & & & & \\
\hline & C. pisifera & ND & $0.936 \dagger$ & 1 & & & & & & & & \\
\hline \multirow[t]{5}{*}{ camphor } & P. densiflora & ND & -0.486 & -0.474 & 1 & & & & & & & \\
\hline & P. koraiensis & ND & -0.390 & -0.424 & 1 & & & & & & & \\
\hline & A .holophylla & $\mathrm{ND}$ & -0.609 & -0.542 & 1 & & & & & & & \\
\hline & C. obtusa & -0.463 & -0.278 & -0.346 & 1 & & & & & & & \\
\hline & C. pisifera & ND & -0.382 & -0.290 & 1 & & & & & & & \\
\hline \multirow[t]{5}{*}{ carene } & P. densiflora & ND & 0.438 & 0.564 & -0.558 & 1 & & & & & & \\
\hline & P. koraiensis & ND & -0.022 & -0.060 & -0.165 & 1 & & & & & & \\
\hline & A .holophylla & $\mathrm{ND}$ & 0.383 & 0.368 & 0.178 & 1 & & & & & & \\
\hline & C. obtusa & -0.160 & $0.775 \dagger$ & $0.936 \dagger$ & -0.289 & 1 & & & & & & \\
\hline & C. pisifera & ND & -0.205 & -0.236 & .007 & 1 & & & & & & \\
\hline \multirow[t]{5}{*}{ eucalyptol } & P. densiflora & ND & -0.285 & -0.261 & $0.721 \dagger$ & -0.331 & 1 & & & & & \\
\hline & P. koraiensis & ND & $\mathrm{ND}$ & ND & ND & ND & 1 & & & & & \\
\hline & A .holophylla & ND & -0.242 & -0.247 & 0.086 & 0.369 & 1 & & & & & \\
\hline & C. obtusa & ND & ND & ND & ND & ND & 1 & & & & & \\
\hline & C. pisifera & ND & -0.504 & -0.315 & -0.131 & -0.401 & 1 & & & & & \\
\hline \multirow[t]{5}{*}{ limonene } & P. densiflora & ND & 0.534 & $0.656^{*}$ & -0.541 & $0.645^{*}$ & -0.061 & 1 & & & & \\
\hline & P. koraiensis & ND & $0.973 \dagger$ & $0.914 \dagger$ & -0.388 & -0.085 & ND & 1 & & & & \\
\hline & A .holophylla & ND & 0.488 & 0.499 & -0.142 & 0.295 & -0.235 & 1 & & & & \\
\hline & C. obtusa & -0.333 & $0.867 \dagger$ & $0.948 \dagger$ & -0.362 & $0.791 \dagger$ & ND & 1 & & & & \\
\hline & C. pisifera & ND & $0.976 \dagger$ & $0.972 \dagger$ & -0.306 & -0.253 & -0.401 & 1 & & & & \\
\hline \multirow[t]{5}{*}{ linalool } & P. densiflora & ND & 0.524 & $0.660^{*}$ & $-0.619 *$ & $0.936 \dagger$ & -0.415 & $0.753 \dagger$ & 1 & & & \\
\hline & P. koraiensis & ND & $0.826 \dagger$ & $0.640^{*}$ & -0.313 & 0.018 & ND & $0.792 \dagger$ & 1 & & & \\
\hline & A .holophylla & ND & $0.715 \dagger$ & $0.622 *$ & -0.380 & $0.578^{*}$ & 0.025 & 0.053 & 1 & & & \\
\hline & C. obtusa & -0.186 & $0.718 \dagger$ & $0.932 \dagger$ & -0.390 & $0.880 \dagger$ & ND & $0.924 \dagger$ & 1 & & & \\
\hline & C. pisifera & ND & $0.961 \dagger$ & $0.883 \dagger$ & -0.427 & -0.035 & -0.507 & $0.930 \dagger$ & 1 & & & \\
\hline \multirow[t]{5}{*}{ myrcene } & P. densiflora & ND & $-0.697^{*}$ & $-0.738 \dagger$ & $0.703^{*}$ & $-0.790 \dagger$ & 0.306 & $-0.742 \dagger$ & $-0.742 \dagger$ & 1 & & \\
\hline & P. koraiensis & ND & -0.573 & $-0.599 *$ & $0.660 *$ & 0.035 & ND & -0.556 & -0.467 & 1 & & \\
\hline & A .holophylla & ND & $-0.577^{*}$ & -0.520 & 0.472 & 0.393 & $0.726 \dagger$ & -0.418 & -0.144 & 1 & & \\
\hline & C. obtusa & 0.353 & -0.569 & $-0.619^{*}$ & 0.367 & -0.388 & ND & $-0.809 \dagger$ & $-0.691^{*}$ & 1 & & \\
\hline & C. pisifera & ND & $-0.828 \dagger$ & $-0.663^{*}$ & 0.183 & 0.088 & $0.720 \dagger$ & $-0.776 \dagger$ & $-0.759 \dagger$ & 1 & & \\
\hline \multirow[t]{5}{*}{ p-cymene } & P. densiflora & ND & -0.103 & 0.070 & -0.156 & $0.683^{*}$ & -0.338 & -0.031 & 0.562 & -0.236 & 1 & \\
\hline & P. koraiensis & ND & 0.430 & 0.299 & -0.108 & 0.123 & ND & 0.407 & $0.847 \dagger$ & -0.260 & 1 & \\
\hline & A .holophylla & ND & 0.519 & 0.426 & 0.036 & $0.893 \dagger$ & 0.301 & 0.316 & 0.570 & 0.141 & 1 & \\
\hline & C. obtusa & -0.093 & $0.769 \dagger$ & $0.936 \dagger$ & -0.286 & $0.987 \dagger$ & ND & $0.793 \dagger$ & $0.895 \dagger$ & -0.372 & 1 & \\
\hline & C. pisifera & $\mathrm{ND}$ & $0.893 \dagger$ & $0.826 \dagger$ & -0.198 & 0.168 & $-0.730 \dagger$ & $0.869 \dagger$ & $0.882 \dagger$ & $-0.827 \dagger$ & 1 & \\
\hline \multirow[t]{5}{*}{ sabinene } & P. densiflora & ND & $0.721 \dagger$ & $0.861 \dagger$ & $-0.597^{*}$ & $0.844 \dagger$ & -0.307 & $0.865 \dagger$ & $0.930 \dagger$ & $-0.828 \dagger$ & 0.317 & 1 \\
\hline & P. koraiensis & ND & $0.812 \dagger$ & 0.435 & -0.353 & 0.213 & ND & $0.678^{*}$ & $0.738 \dagger$ & -0.420 & 0.470 & 1 \\
\hline & A .holophylla & ND & $0.836 \dagger$ & $0.830 \dagger$ & -0.290 & $0.799 \dagger$ & 0.082 & 0.548 & $0.687^{*}$ & -0.125 & $0.799 \dagger$ & 1 \\
\hline & C. obtusa & -0.314 & $0.910 \dagger$ & $0.986 \dagger$ & -0.349 & $0.870 \dagger$ & ND & $0.981 \dagger$ & $0.914 \dagger$ & $-0.708 \dagger$ & $0.867 \dagger$ & 1 \\
\hline & C. pisifera & ND & $0.944 \dagger$ & $0.915 \dagger$ & -0.300 & 0.049 & $-0.592 *$ & $0.947 \dagger$ & $0.930 \dagger$ & $-0.811 \dagger$ & $0.969 \dagger$ & 1 \\
\hline
\end{tabular}

Note: Values are expressed as Pearson's product moment correlation coefficient(n=3), *: p<0.05, $\uparrow: p<0.01)$ 
Table 10. The correlation among the terpenes (2)

\begin{tabular}{|c|c|c|c|c|c|c|c|c|c|c|c|}
\hline & & terpineol & terpinolene & $\alpha$-phellandrene & $\alpha$-pinene & $\alpha$-terpinene & $\beta$-pinene & $\gamma$-terpinene & cedrol & $\begin{array}{c}\text { trans } \\
\text {-caryophyl- } \\
\text { lene } \\
\end{array}$ & $\alpha$-humulene \\
\hline \multirow[t]{5}{*}{ terpineol } & P. densiflora & 1 & & & & & & & & & \\
\hline & P. koraiensis & 1 & & & & & & & & & \\
\hline & A .holophylla & 1 & & & & & & & & & \\
\hline & C. obtusa & 1 & & & & & & & & & \\
\hline & C. pisifera & 1 & & & & & & & & & \\
\hline \multirow[t]{5}{*}{ terpinolene } & P. densiflora & -0.006 & 1 & & & & & & & & \\
\hline & P. koraiensis & 0.413 & 1 & & & & & & & & \\
\hline & A .holophylla & 0.214 & 1 & & & & & & & & \\
\hline & C. obtusa & $0.670^{*}$ & 1 & & & & & & & & \\
\hline & C. pisifera & -.0444 & 1 & & & & & & & & \\
\hline \multirow[t]{5}{*}{$\alpha$-phellandrene } & P. densiflora & 0.454 & 0.328 & 1 & & & & & & & \\
\hline & P. koraiensis & 0.182 & 0.338 & 1 & & & & & & & \\
\hline & A .holophylla & 0.532 & $0.776 \dagger$ & 1 & & & & & & & \\
\hline & C. obtusa & 0.200 & $0.629 *$ & 1 & & & & & & & \\
\hline & C. pisifera & -0.515 & $0.716 \dagger$ & 1 & & & & & & & \\
\hline \multirow[t]{5}{*}{$\alpha$-pinene } & P. densiflora & 0.077 & $0.862 \dagger$ & 0.459 & 1 & & & & & & \\
\hline & P. koraiensis & 0.362 & $0.742 \dagger$ & $0.848 \dagger$ & 1 & & & & & & \\
\hline & A .holophylla & 0.263 & $0.970 \dagger$ & $0.786 \dagger$ & 1 & & & & & & \\
\hline & C. obtusa & $0.589 *$ & $0.953 \dagger$ & $0.824 \dagger$ & 1 & & & & & & \\
\hline & C. pisifera & -0.304 & $0.857 \dagger$ & 0.431 & 1 & & & & & & \\
\hline \multirow[t]{5}{*}{$\alpha$-terpinene } & P. densiflora & 0.448 & 0.448 & $0.962 \dagger$ & $0.632^{*}$ & 1 & & & & & \\
\hline & P. koraiensis & 0.151 & 0.348 & $0.998 \dagger$ & $0.845 \dagger$ & 1 & & & & & \\
\hline & A .holophylla & 0.417 & $0.899 \dagger$ & $0.961 \dagger$ & $0.867 \dagger$ & 1 & & & & & \\
\hline & C. obtusa & $0.585^{*}$ & $0.978 \dagger$ & $0.772 \dagger$ & $0.990 \dagger$ & 1 & & & & & \\
\hline & C. pisifera & -0.537 & $0.955 \dagger$ & $0.838 \dagger$ & $0.816 \dagger$ & 1 & & & & & \\
\hline \multirow[t]{5}{*}{$\beta$-pinene } & P. densiflora & 0.078 & $0.913 \dagger$ & 0.485 & $0.988 \dagger$ & $0.642 *$ & 1 & & & & \\
\hline & P. koraiensis & 0.250 & 0.516 & $0.972 \dagger$ & $0.948 \dagger$ & $0.969 \dagger$ & 1 & & & & \\
\hline & A .holophylla & 0.405 & $0.871 \dagger$ & $0.854 \dagger$ & $0.952 \dagger$ & $0.861 \dagger$ & 1 & & & & \\
\hline & C. obtusa & 0.511 & $0.907 \dagger$ & $0.895 \dagger$ & $0.990 \dagger$ & $0.970 \dagger$ & 1 & & & & \\
\hline & C. pisifera & -0.397 & $0.955 \dagger$ & $0.635^{*}$ & $0.963 \dagger$ & $0.937 \dagger$ & 1 & & & & \\
\hline \multirow[t]{5}{*}{$\gamma$-terpinene } & P. densiflora & 0.342 & $0.650^{*}$ & $0.854 \dagger$ & $0.801 \dagger$ & $0.951 \dagger$ & $0.821 \dagger$ & 1 & & & \\
\hline & P. koraiensis & 0.105 & $0.612 *$ & $0.753 \dagger$ & $0.719 \dagger$ & $0.782 \dagger$ & $0.751 \dagger$ & 1 & & & \\
\hline & A .holophylla & 0.319 & $0.983 \dagger$ & $0.876 \dagger$ & $0.955 \dagger$ & $0.962 \dagger$ & $0.893 \dagger$ & 1 & & & \\
\hline & C. obtusa & $0.630^{*}$ & $0.996 \dagger$ & $0.689^{*}$ & $0.971 \dagger$ & $0.992 \dagger$ & $0.936 \dagger$ & 1 & & & \\
\hline & C. pisifera & -0.516 & $0.986 \dagger$ & $0.776 \dagger$ & $0.849 \dagger$ & $0.986 \dagger$ & $0.957 \dagger$ & 1 & & & \\
\hline \multirow[t]{5}{*}{ cedrol } & P. densiflora & -0.050 & $0.854 \dagger$ & 0.187 & $0.860 \dagger$ & 0.381 & $0.844 \dagger$ & 0.566 & 1 & & \\
\hline & P. koraiensis & -0.082 & 0.434 & $0.853 \dagger$ & $0.828 \dagger$ & $0.866 \dagger$ & $0.886 \dagger$ & $0.697^{*}$ & 1 & & \\
\hline & A .holophylla & -0.008 & $0.0853 \dagger$ & $0.693^{*}$ & $0.899 \dagger$ & $0.763 \dagger$ & $0.867 \dagger$ & $0.833 \dagger$ & 1 & & \\
\hline & C. obtusa & $0.730 * *$ & $0.972 \dagger$ & $0.650 *$ & $0.947 \dagger$ & $0.951 \dagger$ & $0.909 \dagger$ & $0.967 \dagger$ & 1 & & \\
\hline & C. pisifera & -0.331 & $0.806 \dagger$ & $0.604^{*}$ & $0.667^{*}$ & $0.768 \dagger$ & $0.749 \dagger$ & $0.757 \dagger$ & 1 & & \\
\hline trans & P. densiflora & 0.050 & $0.970 \dagger$ & 0.449 & $0.841 \dagger$ & 0.545 & $0.911 \dagger$ & $0.730 \dagger$ & $0.740 \dagger$ & & \\
\hline \multirow[t]{4}{*}{-caryophyllene } & P. koraiensis & 0.129 & $0.747 \dagger$ & $0.814 \dagger$ & $0.897 \dagger$ & $0.832 \dagger$ & $0.874 \dagger$ & $0.916 \dagger$ & $0.808 \dagger$ & 1 & \\
\hline & A .holophylla & 0.250 & $0.733 \dagger$ & $0.604^{*}$ & $0.836 \dagger$ & $0.632^{*}$ & $0.889 \dagger$ & $0.707^{*}$ & $0.808 \dagger$ & 1 & \\
\hline & C. obtusa & $0.682^{*}$ & $0.889 \dagger$ & 0.540 & $0.827 \dagger$ & $0.842 \dagger$ & $0.794 \dagger$ & $0.873 \dagger$ & $0.945 \dagger$ & 1 & \\
\hline & C. pisifera & -0.379 & $0.910 \dagger$ & $0.654^{*}$ & $0.663^{*}$ & $0.813 \dagger$ & $0.787 \dagger$ & $0.850 \dagger$ & $0.908 \dagger$ & 1 & \\
\hline \multirow[t]{5}{*}{$\alpha$-humulene } & P. densiflora & 0.062 & $0.971 \dagger$ & 0.473 & $0.848 \dagger$ & 0.574 & $0.915 \dagger$ & $0.755 \dagger$ & $0.759 \dagger$ & 1 & 1 \\
\hline & P. koraiensis & 0.145 & $0.760 \dagger$ & $0.797 \dagger$ & $0.883 \dagger$ & $0.817 \dagger$ & $0.856 \dagger$ & $0.929 \dagger$ & $0.781 \dagger$ & $0.998 \dagger$ & 1 \\
\hline & A .holophylla & 0.227 & $0.589 *$ & 0.541 & $0.734 \dagger$ & 0.524 & $0.842 \dagger$ & 0.574 & $0.753 \dagger$ & $0.998 \dagger$ & 1 \\
\hline & C. obtusa & -0.181 & 0.205 & 0.495 & 0.359 & 0.300 & 0.395 & 0.252 & 0.232 & $0.973 \dagger$ & 1 \\
\hline & C. pisifera & -0.548 & 0.427 & $0.861 \dagger$ & 0.205 & $0.655^{*}$ & 0.393 & 0.554 & 0.275 & 0.088 & 1 \\
\hline
\end{tabular}

Note: Values are expressed as Pearson's product moment correlation coefficient(n=3), *: $<<0.05, \uparrow: p<0.01)$

relations with bornyl acetate, camphene, carene, limonene, linalool, $\alpha$-pinene, $\alpha$-terpinene, $\beta$-pinene, and $\gamma$-terpinene, camphor showed negative $(-)$ correla- tions with $\gamma$-terpinene, and myrcene and camphor showed positive $(+)$ correlations with each other. In the essential oil extracted from the leaves of Pinus koraien- 
sis, myrcene showed negative (-) correlations with camphene, terpinolene, $\alpha$-pinene, $\gamma$-terpinene, trans-caryophyllene, and $\alpha$-humulene and in the essential oil extracted from the leaves of Abies holophylla, myrcene showed negative (-) correlations with bornyl acetate and trans-caryophyllene and camphor showed negative (-) correlations with bornyl acetate. In the essential oil extracted from the leaves of Chamaecyparis obtuse, myrcene showed negative (-) correlations with camphene, limonene, linalool, sabinene, terpinolene, $\alpha-$ pinene, $\alpha$-terpinene, $\gamma$-terpinene, cedrol, and trans-caryophyllene. In the essential oil extracted from the leaves of Chamaecyparis pisifera, myrcene showed negative (-) correlations with bornyl acetate, camphene, limonene, linalool, p-cymene, sabinene, terpinolene, $\alpha$ phellandrene, $\alpha$-pinene, $\alpha$-terpinene, $\beta$-pinene, $\gamma$-terpinene, cedrol, and trans-caryophyllene, eucalyptol showed negative (-) correlations with p-cymene, sabinene, $\alpha$-phellandrene, $\alpha$-terpinene, $\gamma$-terpinene, transcaryophyllene, and $\alpha$-humulene, and myrcene and eucalyptol showed positive $(+)$ correlations with each other.

Zafra and García-Peregrín (1976) reported that in the essential oil extracted from the leaves of Pinus syvestris, the contents of sabinene and camphene were inversely proportional to each other, but in the present study, the results of analyses of Pinus densiflora and Pinus koraiensis, which are in the same genus as Pinus syvestris, were different.

The reason why the results of the present study are partially inconsistent with the previous studies is thought to be that the tree species studied were different. Although the genus was the same, individuals have genetic differences from each other, even if they are in the same species, and terpenic compounds are subject to genetic influences (Pauly and Rudloff, 1971; Franklin, 1976). In addition, even the same individual contains different terpenic compounds among the branches or locations where samples were collected (Rudloff, 1972; Rudloff and Granat, 1982; Sim and Ahn, 1989) and the contents of terpenic compounds are different among growth and development environments, as well (Wilkinson et al., 1971; Son and Hwang, 1990). In addition, various enzymes involved in the biosynthesis of essential oil in mevalonate pathways, which are biosynthesis metabolism paths, are affected by seasonal changes, temperatures, and photosynthesis (Abad Farooqi et al., 1993), and the damage by diseases and insects or pests suffered during growth and development can also be a cause of the differences (Wilkinson, 1980; Harris et al., 1983).

Because changes in the concentrations of terpenic compounds are affected by diverse factors as such, continued studies are necessary to establish correlations. However, if essential oils are to be extracted from the leaves of coniferous trees and used in aromatherapy, flavoring agents, or deodorizing agents, according to the results of studies thus far, extracting essential oils in spring and summer when the concentrations of terpenic compounds are high is judged to be most effective.

\section{CONCLUSTIONS}

The present study was conducted to compare the contents of various terpenic compounds contained in essential oils extracted from the leaves of five species of coniferous trees to identify tree species that contain relatively high concentrations of terpenic compounds and determine seasonal changes in the concentrations of the terpenic compounds. In addition, the present study was also intended to compare terpenic compounds contained in the essential oils and those that are volatilized from the leaves through stomas to determine whether the terpenic compounds contained in the leaves actually volatilize to the air.

According to the results, first, the concentrations of terpenic compounds were relatively lower in essential oils extracted from the leaves of Pinus densiflora and Pinus koraiensis compared to Abies holophylla, Chamaecyparis obtusa, and Chamaecyparis pisifera. In addition, the concentrations of eight kinds of terpenic compounds in the leaves of Abies holophylla, nine kinds in the leaves of Chamaecyparis obtuse, and three kinds in the leaves of Chamaecyparis pisifera were particularly higher compared to other tree species. However, as for the concentrations of useful terpenic compounds volatilized through stomas in airtight containers, eights kinds in the leaves of Pinus densiflora, 16 kinds in the leaves of Pinus koraiensis, 10 kinds in the leaves of Abies holophylla, one kind in the leaves of Chamaecyparis obtuse, and two kinds in the leaves of Chamaecyparis pisifera showed relatively higher concentrations compared to other tree species. Therefore, woodland walks or activities for forest healing in Pinus densiflora, Pinus koraiensis, or Abies holophylla forests are judged very effective.

Second, the seasonal total quantities of the 21 kinds of useful terpenic compounds contained in the essential oils extracted from the five species of coniferous trees were large in summer in the case of Abies holophylla and in spring in the cases of Pinus densiflora, Pinus koraiensis, Chamaecyparis obtusa, and Chamaecyparis pisifera. In addition, the concentrations of various kinds of terpenic compounds by kind were found to be high in spring and summer through analyses. However, the concentration of myrcene was shown to commonly increase as seasons changed from spring to summer, autumn, and winter in order of precedence in the essential oils extracted from the leaves of the five species of coniferous trees and showed negative (-) correlations with most terpenic compounds. Therefore, if essential oils are to be extracted from the leaves of coniferous trees and utilized in forest healing, extracting essential oils in spring and summer when the concentrations of terpenic compounds are high is judged most effective.

\section{AUTHOR CONTRIBUTIONS}

C. S. SHIN designed the study and wrote the paper. J. W. LEE and P. S. YEON performed the chemical analy- 
sis. C. S. SHIN and J. Y. CHA performed data analysis. P. S. YEON and S. OHGA designed the study, supervised the work. All authors assisted in editing the manuscript and approved the final version.

\section{ACKNOWLEDGMENTS}

This study was carried out with the support of 'Forest Science \& Technology Projects (Project No. S211214L010110)' provided by Korea Forest Service.

\section{REFERENCES}

Abad Farooqi, A. H., Sharma, S., Naqvi, A. A. and A. Khan 1993 The effect of kinetin on flower and oil production in rosa damascena. Journal of Essential Oil Research, 5: 305-309

Bakkali, F., Averbeck, S., Averbeck, D., and M. Idaomar 2008 Biological effects of essential oils - a review. Food and Chemical Toxicology, 46: 446-475

Franklin, E. C. 1976 Within-tree variation of monoterpene composition and yield in slash pine clones and families. Forest Science, 22: 185-191

Gwak, K. S., Park, M. J., Jeung, E. B., Chang, J. W. and I. G. Choi 2006 Comparison of antifungal activities of monoterpenes and sesquiterpenes in essential oil from chamaecyparis obtusa against dermatophytes. Journal of The Korean Wood Science and Technology, 34: 46-55

Harris, L. J., Borden, J. H., Pierce Jr, H. D. and A. C. Oehlschlager 1983 Cortical resin monoterpenes in Sitka spruce and resistance to the white pine weevil, Pissodes strobi (Coleoptera: Curculionidae). Canadian Journal of Forest Research, 13: 350-352

Kang, S. K., Shin, M. K., Auh, K. S., Chun, Y. H. and J. P. Hong 2007 Antibacterial effect on oral pathogenic bacteria of phytoncide from chamaecyparis obtusa. Korean Journal of Oral Medicine, 32: 45-55

Kim, H. S., Han, S. K. and J. Y. Mang 2009 Evaluations on the deodorization effect and antibacterial activity of chamaecyparis obtusa essential oil. Korean Journal of Odor Research and Engineering, 8: 111-117

Kim, J. C. 2001 Factors controlling natural VOC emissions in a southeastern US pine forest. Atmospheric Environment, $\mathbf{3 5}$ : 3279-3292

Kim, K. W. 2014 Relationship between seasonal NVOC concentration and physical environment in pinus densiflora forest. Chungnam National University, Daejeon, Republic of Korea

Kim, S. H., Kim, D. Y., Lee, J. S., Jung, S. W., Park, S. H., Yoo, B. K., Park, H. J. and K. Y. Byeon 2011 In A study on the seasonal occurrence characteristics phytoncide in Ulsan, Proceedings of the Korean Environmental Sciences Society Conference, 231232

Korea Forest Service 2014 Cypress forest air is highly effective in the treatment of asthma. http://www.forest.go.kr/newkfsweb/ cop/bbs/selectBoard List.do?bbsId=BBSMSTR_1036\&mn= KFS_03_02 (accessed on 24 June 2014)

Korea Forest Service 2014 Atopy treatment substance found in cypress leaf essential oil. http://www.forest.go.kr/newkfsweb/ cop/bbs/selectBoardList. do?bbsId=BBSMSTR_1036\&mn= KFS_03_02 (accessed on 25 June 2014)

Kubeczka, K. H. and W. Schultze 1987 Biology and chemistry of conifer oils. Flavour and Fragrance Journal, 2: 137-148

Lee, H. E., Shin, W. S., Yeoun, P. S., Cho, Y. M. and S. H. Yeom 2011 Effects of inhalation of essential oil (chamaecyparis obtusa) on stress and depression in college students. The Journal of Korean Institute of Forest Recreation, 15: 61-68

Lee, H. O., Baek, S. H. and D. M. Han 2001 Antimicrobial effects of chamaecyparis obtusa essential oil. Korean Journal of Microbiology and Biotechnology, 29: 253-257
Lee, K. K. 1999 Antimicrobial activity of thuja orientalis and chamaecyparis obtusa essential oil. Korean Beauty Society, $\mathbf{5}$ : $567-577$

Mishra, A. K. and N. K. Dubey 1994 Evaluation of some essential oils for their toxicity against fungi causing deterioration of stored food commodities. Applied and Environmental Microbiology, 60: 1101-1105

Moon, B. H. and K. S. Yoo 2005 Characterization of volatile essential oil from needle leaves by gas chromatography-mass spectrometry. Journal of the Korea Society for Environmental Analysis, 8: 181-185

Na, K. J., Kang, H. Y., Yoon, S. K., Choi, I. G., Yun, Y. W. and E. B. Jeung 1998 The sedative effect of stress by essential oils purifed from softwoods. Laboratory Animal Research, 14: 93-96

Na, K. J., Kang, H. Y., Yoon, S. K. and E. B.Jeung 1999 Biological efficacy of essential oils from softwood. Laboratory Animal Research, 15: 79-81

Oh, K. Y., Seo, Y. K., Park, K. H., Kim, I. S., Bae, J. S., Park, S. I., Ha, H., Yang, S. I., Yang, S. I. and W. J. Lee 2013 Comparison of monoterpene in ambient air at forest and essential oil. Journal of Korean Forest Society, 102: 309-314

Park, M. J., Choi, W. S., Min, B. C., Kim, H. Y., Kang, H. Y. and I. K. Choi 2008 Antioxidant activities of essential oils from chamaecyparis obtusa. Journal of the Korean Wood Science and Technology, 36: 159-167

Park, M. J., Lee, S. M., Gwak, K. S., Jeung, E. B., Chang, J. W. and I. K. Choi 2005 Investigation of active antifungal compounds of essential oil from chamaecyparis obtusa against dermatophytes, microsporum canis and trichophyton mentagrophytes. Journal of the Korean Wood Science and Technology, 33: 72-78

Park, Y. O., Kim, Y. C. and B. S. Chang 2013 Hair growth effect of chamaecyparis obtusa oil in c57bl/6 mice. Journal of Investigative Cosmetology, 9: 87-95

Pauly, G. and E. V. Rudloff 1971 Chemosystematic studies in the genus pinus: The leaf oil of pinus contorta var.. Latifolia. Canadian, 49: 1201-1210

Rudloff, E. V. 1972 Seasonal variation in the composition of the volatile oil of the leaves, buds, and twigs of white spruce (picea glauca). Canadian Journal of Botany, 50: 1595-1603

Rudloff, E. V. and M. Granat 1982 Seasonal variation of the terpenes of the leaves, buds, and twigs of balsam fir (abies balsamea) Canadian Journal of Botany, 60: 2682-2685

Shin, W. S., Kim, S. K., Yeon, P. S. and J. H. Lee 2010 Effects of phytoncides on psychophysical responses. The Journal of Korean Institute of Forest Recreation, 14: 85-91

Sim, K. S. and Y. W. Ahn 1989 Domestic production and utilization of oleoresin (2) - seasonal variation of monoterpene composition in needles of Korean white pine (Pinus koraiensis S. et Z). Journal of the Korean Forestry Energy Resources, 9: 35-48

Son, J. O. and B. H. Hwang 1990 Terpenoid analysis of the main softwoods essential ol - comparision of terpenoid components in season. Forest Bioenergy, 10: 84-96

Son, J. O. and B. H. Hwang 1990 Terpenoid analysis of the main softwoods essential ol - comparision of terpenoid components in softwood. Forest Bioenergy, 10: 97-106

Song, H. K. 1995 Biogenetic differences in essential oil constituents from the leave of pinus densiflora and pinus koraiensis. Journal of the Korean Wood Science and Technology, 23 49-53

Wilkinson, R. C. 1980 Relationship between cortical monoterpenes and susceptibility of eastern white pine to white-pine weevil attack. Forest Science, 26: 581-589

Wilkinson, R. C., Hanover, J. W., Wright, J. W. and R. H. Flake 1971 Genetic variation in the monoterpene composition of white spruce. Forest Science, 17: 83-90

Zafra, M. and E. García-Peregrín 1976 Seasonal variations in the composition of pinus halepensis and pinus sylvestris twigs and needles essential oil. The Journal of Agricultural Science, $\mathbf{8 6}$ $1-6$ 